\title{
Inferring Hospital Quality from Patient Discharge Records Using a Bayesian Selection Model
}

\author{
John Geweke \\ Departments of Economics and Statistics \\ University of Iowa \\ John-geweke@uiowa.edu \\ Gautam Gowrisankaran \\ Department of Economics \\ University of Minnesota \\ gautam@econ.umn.edu \\ Robert J. Town \\ Graduate School of Management \\ University of California-Irvine \\ rjtown@uci.edu
}

January 17,2000

\begin{abstract}
This paper develops new econometric methods to estimate hospital quality and other models with discrete dependent variables and non-random selection. Mortality rates in patient discharge records are widely used to infer hospital quality. However, hospital admission is not random and some hospitals may attract patients with greater unobserved severity of illness than others. In this situation the assumption of random admission leads to spurious inference about hospital quality. This study controls for hospital selection using a model in which distance between the patient's residence and alternative hospitals are key exogenous variables. Bayesian inference in this model is feasible using a Markov chain Monte Carlo posterior simulator, and attaches posterior probabilities to quality comparisons between individual hospitals and groups of hospitals. The study uses data on 77.937 Medicare patients admitted to 117 hospitals in Los Angeles County from 1989 through 1992 with a diagnosis of pneumonia. It finds higher quality in smaller hospitals than larger, and in private for-profit hospitals than in hospitals in other ownership categories. Variations in unobserved severity of illness across hospitals is at least a great as variation in hospital quality. Consequently a conventional probit model leads to inferences about quality markedly different than those in this study's selection model.
\end{abstract}

We thank Pat Bajari, Lanier Benkard, Mike Chernew, Tom Holmes, Steven Stern and seminar participants at Princeton University, the University of Michigan, the University of Virginia, and the Society of Economic Dynamics 1999 Annual Meetings for helpful comments. The first author acknowledges support from NSF grant SBR-9819444 and the second author acknowledges support from the University of Minnesota Supercomputer Institute. 


\section{Introduction}

This paper develops new econometric methods to estimate hospital quality and other models with discrete dependent variables and non-random selection. Assessing the quality of care in hospitals is an important problem for public policy and a challenge for applied econometrics. ${ }^{1}$ Policy changes in Medicare reimbursement rates and the rise of managed care as well as technological innovations have affected hospital incentives, and through that, hospital quality. ${ }^{2}$ These quality changes have large welfare effects and hence the potential for large deadweight losses. $^{3}$

Hospital patient discharge databases provide several indicators plausibly associated with hospital quality. Since they cover large numbers of patients and hospitals and are much less expensive to obtain and access than other sources of information, they have been widely used in comparisons of hospital quality. Mortality has been the most popular indicator of hospital quality in the literature: it is unambiguously defined and free of measurement error, and its link with quality of care is so strong as to be tautological. ${ }^{4}$

In this widely used framework, the conceptual experiment that reveals hospital quality is hospital-specific mortality rates following random assignment of a population of patients to hospitals. Patients, however, are not randomly assigned to hospitals. Patients or their physicians are likely to choose hospitals based on factors such as location, convenience and their severity of illness. Econometrically, the experiment implicit in the data is not random assignment, and the corresponding real experiment of random patient assignment cannot be performed. If assignment were nonrandom, but random conditional on observed characteristics, then conventional dichotomous outcome models could be used to infer the outcome of the conceptual experiment from the available data. However, discharge data contain only crude summaries of medically pertinent information and hence many aspects of the severity of illness are unobserved. Thus, the

\footnotetext{
1 "As described by a leading study, "Quality of care is the degree to which health services for individuals and populations increase the likelihood of desired health outcomes and are consistent with current professional knowledge...," Lohr (1990, p. 4).

${ }^{2}$ See Cutler (1995), Kessler and McClellan (2000), McClellan and Noguchi (1998) for studies on the effects of Medicare policy, the impact of managed care and the impacts of technological change on medical outcomes, respectively.

${ }^{3}$ For instance, if changes in Medicare policies cause hospitals to reduced their pneumonia mortality rates by one percent, this would translate to over 6,000 lives saved annually in the U.S.

${ }^{4}$ Strictly speaking mortality is an indicator of hospital mediocrity; mortality is an inverse indicator of quality. Subsequently we provide a precise definition of hospital quality in the context of the model developed in this study.
} 
assumption of conditional randomness is not tenable and patients with the same observed characteristics are not equally likely to be admitted to all hospitals. For instance, if patients with high unobserved severity of illness select high quality hospitals, then observed mortality rates for high quality hospitals will be inconsistent and upwardly biased measures of mortality from the conceptual experiment. This will be true even after controlling for observed measures of severity of illness. Conventional statistical methods that ignore unobserved severity will produce misleading inferences about hospital quality. This has led prominent medical experts to make a pessimistic assessment of the usefulness of discharge data in assessing hospital quality. ${ }^{5}$

Recent work by Gowrisankaran and Town (1999) developed a framework to control for the non-random assignment of patients. This work modeled mortality as a function of indicator variables for each hospital and patient discharge information. The authors treat mortality as continuous, and directly apply linear instrumental variables methods. The identifying assumption is that unobserved patient severity is identically distributed in the population. Then, distance to a given hospital is correlated with choice of hospital but uncorrelated with unobserved severity of illness, making it an appropriate instrument for the endogenous choice of hospital. Conceptually, the estimator would predict hospital A to be of higher quality than hospital B if patients residing near hospital A have lower mortality, after controlling for their medical and demographic characteristics. The size of the quality difference between hospitals A and B would be inversely related to the distance that patients are willing to travel to seek care.

However, the outcome variable mortality is dichotomous. Thus, any internally consistent model of hospital quality and choice must be nonlinear. Conventional instrumental variables methods have heretofore not been applied to nonlinear models with non-random assignment. ${ }^{6}$ This paper develops a logically coherent model designed to infer the outcome of the conceptual experiment that randomly assigns patients to hospitals, given data that has non-random patient assignment. Estimating this model is difficult because the amount of information per observation is small and the signal to noise ratio is likely to be small as well. ${ }^{7}$ This paper develops an approach to inference in this model that is practical with the large data sets required to extract

\footnotetext{
${ }^{5}$ Leading medical researchers, including Iezzoni et al. (1996)., and government studies (US GAO (1994)) have both argued that discharge databases are problematic, for this reason.

${ }^{6}$ Though the methods of Gowrisankaran and Town (1999) are much simpler than the ones developed in this paper, there is no formal statistical model that rationalizes their approach.

${ }^{7}$ Simple measures of fit always indicate that most variation in mortality cannot be ascribed to covariates. Even if all the difference in mortality rates were attributable to quality, the variation in these rates is small.
} 
signal from noise in hospital patient discharge databases. This approach, defined by these two methodological advances, is potentially applicable to a wide range of policy evaluations of economic interest where the outcome variable is dichotomous. ${ }^{8}$

The model developed here incorporates hospital choice and mortality as endogenous variables, and fixed hospital and patient characteristics as exogenous variables. Hospital choice is described by a multinomial probit model, and mortality by a binary probit model. The mortality model includes indicator variables for each hospital to accommodate hospital specific differences in quality. It is structural, in the sense that it predicts outcomes for any arbitrary assignment of patients to hospitals, including random assignment. The multinomial probit model is a reduced form relationship that provides probabilities of hospital choice conditional on observed covariates that are a function of demographic characteristics and distance of the hospital from the patient's home. The random component in the binary probit model includes unobserved severity of illness, and is permitted to be correlated with the random component in the multinomial choice model. Thus, the model accommodates the possibility that the greater a patient's probability of mortality due to unobserved severity, the more likely it is that the patient is admitted to some hospitals rather than others.

The methodology developed here exploits the similarity between this model and the conventional linear simultaneous equation model. Were the latent utility in this model fully observed, the mortality probit equation would be a linear structural equation, and the hospital choice multinomial probit equations would be the reduced form of the remainder of the model. Only the appearance of the discrete hospital choice in the mortality probit equation would depart from the classical specification that gives rise to instrumental variable methods. The model handles the unobserved nature of the latent variables through the use of Bayesian simulation methods. These methods iteratively simulate latent variable values conditional on the data, and parameters conditional on the latent variables. The discrete hospital choice in the mortality probit equation does not pose a problem, and hence the second step is computationally similar to classical instrumental variables. In this way, the simulation methods simultaneously recover the joint posterior distribution of parameters and latent variables. ${ }^{9}$ By transforming the problem from

\footnotetext{
${ }^{8}$ Examples include the effect of school performance based on graduation rates, of prison rehabilitation programs based on recidivism rates, of job training programs based on the incidence of harassment complaints, and many medical outcome evaluations.

${ }^{9}$ Surveys that discuss convergence include Chib and Greenberg (1996), Geweke (1996) and Geweke (1999).
} 
an integration problem into a Markov chain Monte Carlo simulation problem, the methodology developed here can be used to compute estimates orders of magnitude faster than the method of maximum likelihood. ${ }^{10}$ This makes it feasible to estimate this type of simultaneous equations model. Albert and Chib (1993) applied these methods to the binary probit model and Geweke, Keane and Runkle (1997) extended them to the multinomial probit model. The methods developed here extend this approach to a new class of models.

In addition to handling the latent variable problem, Bayesian inference makes it possible to address the motivating policy questions directly, by providing marginal posterior distributions for any functions of parameters. These functions include the probability of mortality in the conceptual random assignment experiment, and the posterior probability that this mortality rate is lower for one hospital than for another.

The data used in this study are taken from the hospital discharge records of 77,937 Medicare patients admitted to 117 hospitals in Los Angeles County during 1989 through 1992 with a diagnosis of pneumonia. The discharge records contain demographic information, including patient addresses, and summary measures of severity of illness at the time of admission. The address data is used to construct the distance of each patient's home from each hospital. Functions of this distance variable, alone and in combination with demographic characteristics, play a role in the model analogous to that played by the instrumental variables in coping with endogeneity in linear models. The large size of the data set is essential because of the low signalto-noise problem: the ratio of patients to hospitals is roughly 660, but many hospitals treated fewer than 300 patients, and the overall mortality rate is .095 . The number of latent variables is roughly the product of the number of patients and number of hospitals, on the order of $10^{7}$, making this one of the largest models of its kind ever applied. This places a premium on issues of computational efficiency, addressed in this study.

Conditional on this data set, the posterior distribution for the parameters of the model has a number of interesting substantive implications. There is substantial variance to the posterior distribution of quality of most individual hospitals: for about 70 percent of the hospitals, there is a posterior probability of at least 10 percent that the hospital is in one of each of three quartiles

\footnotetext{
${ }^{10}$ Maximum likelihood evaluation for one parameter vector for one individual would require evaluating the joint density of the mortality and hospital choice outcome for that individual. Given that the mortality error and hospital choice error are correlated, this would take several minutes on a fast supercomputer. Multiplied by a data set of
} 
of the quality distribution. Nonetheless, there appear to be two key relations between hospital characteristics and quality. Hospitals that are small- fewer than 150 beds-have lower mortality than larger hospitals, and private for-profit hospitals have lower mortality rates than public, teaching, and private not-for-profit hospitals. Turning to the process of hospital admission, there is strong evidence that the level of unobserved severity of illness differs across hospitals. For some hospitals, a high level of unobserved severity increases the probability that a patient will be admitted to that hospital, while for others it decreases the probability of admission.

Unobserved severity of illness is found to be positively correlated with estimated hospital quality. This variation in selectivity turns out to be at least as important as variation in hospital quality in explaining variation in mortality rates across hospitals conditional on observed patient characteristics. The simple probit model attributes all variation in hospital mortality rates, conditional on observed characteristics, to hospital quality differences, and therefore leads to strikingly different conclusions about comparative quality. While there is substantial variation in between the quality, probit quality and severity relationship across hospitals, two generalizations can be made. First, the simple probit model overstates the variation in quality differences, because of the large variation in selectivity. Second, unlike the selection model, the simple model does not reveal any sharp relations between hospital characteristics and quality.

Section 2 provides the specification of the model and methods for inference, with some details relegated to an appendix. The database is described in Section 3. Section 4 presents findings on hospital quality and the role of nonrandom admission to hospitals. Section 5 concludes by addressing some of the questions that motivated the work. An Appendix details the likelihood function, posterior density, Gibbs sampling algorithm and computational time.

roughly 80,000 patients (necessary because of the small signal to noise ratio), it would take months to evaluate the likelihood for a single parameter vector. 


\section{The model}

The key component of the model is a structural probit equation, in which the probability of mortality is a function of the hospital to which a patient is admitted, the observed severity of the patient's illness, and the observed demographic characteristics of the patient. The objective is to learn about the way the hospital to which the patient is admitted influences the probability of mortality in this equation. A multinomial probit model of hospital admission supplements the mortality model, to permit non-random assignment of patients to hospitals. This section describes, in turn, the specification of the model, the prior distribution of the model parameters, and methods to recover the posterior distribution of these parameters.

\subsection{Model specification}

Let $i=1, \ldots, n$ index the patients in the sample, and let $j=1, \ldots, J$ index hospitals in the sample. There are three groups of exogenous variables in the model. The $k \times 1$ vector $\mathbf{x}_{i}$ consists of individual characteristics of patient $i$ that may affect mortality, including indicators for age, race, sex, and disease stage, and measures of income. The specifics of these variables are presented in Section 3. The $q \times 1$ vector $\mathbf{z}_{i j}$ consists of characteristics specific to the combination of individual $i$ and hospital $j$. The variables in $\mathbf{z}_{i j}$ are the distance between the home of patient and hospital $j$, the square of this distance, and the products of distance and age, disease stage, and income, respectively; $q=5$. The $r \times 1$ vector $\mathbf{w}_{i}$ consists of class membership indicators for hospital $i$. The first class is universally inclusive, so the first element of $\mathbf{w}_{i}$ is always 1 ; there are four classes for hospital size and four for ownership status as detailed in Section 3; and there is a class specific to each of the $J$ hospitals. Thus $r=J+9$.

There are two sets of endogenous variables in the model. The mortality indicator $m_{i}$ is 1 if the patient dies in the hospital within ten days of admission and is 0 otherwise. The $J \times 1$ indicator vector $\mathbf{c}_{i}$ has $j$ 'th entry 1 if patient $i$ is admitted to hospital $j$, and is 0 otherwise.

To present the structural mortality equation, define the $J \times r$ matrix $\mathbf{W}$ with $i$ 'th row $\mathbf{w}_{i}^{\prime}$,

and let $\varepsilon_{i}(i=1, \ldots, n)$ be independent $\mathrm{N}\left(0, \sigma^{2}\right)$ random variables conditional on the exogenous variables. The mortality probit $m_{i}^{*}$ is a latent random variable,

$$
m_{i}^{*}=\mathbf{c}_{i}^{\prime} \mathbf{W} \beta+\mathbf{x}_{i}^{\prime} \gamma+\varepsilon_{i}
$$


The mortality indicator $m_{i}=1$ if $m_{i}^{*}>0$ and $m_{i}=0$ if $m_{i}^{*} \leq 0$. The structural interpretation of (1) is that if patient $i$ were randomly assigned to hospital $j$, then $m_{i}^{*}=\mathbf{w}_{j}^{\prime} \beta+\mathbf{x}_{i}^{\prime} \gamma+\varepsilon_{i}$ and consequently $\mathrm{P}\left(m_{i}=1\right)=\Phi\left[\left(\mathbf{w}_{j}^{\prime} \beta+\mathbf{x}_{i}^{\prime} \gamma\right) / \sigma\right]$. Because $J>r, \beta$ is not identified in the classical sense: one could add a constant to $\beta_{j}$ for some $j \leq r-J$, and subtract the same constant from $\beta_{i}(i>r-J)$ for all those hospitals in the class indicated by element $j$ of $\mathbf{w}_{i}$. The vector $\beta$ is not identified because (1) remains structural given all such changes. We construct $\mathbf{W}$ and $\beta$ in this way because it facilitates the development of the prior distribution subsequently in Section 2.2. The parameters $\beta$ and $\sigma$ are also jointly unidentified in (1) because they can be scaled by the same arbitrary positive constant without changing the behavior of $m_{i}$. In the conventional probit model this problem is avoided by setting $\sigma=1$. We return to this matter in the context of the complete model below.

If $\mathbf{c}_{i}$ were in fact independent of $\varepsilon_{i}$ - as it would be if patients were randomly assigned to hospitals, for example - then $\mathbf{c}_{i}^{\prime} \mathbf{W}$ would be exogenous in (1). After resolution of identification issues this model would conform with the conventional textbook specification of the binary probit model. However, it is likely that in observed data, $\mathbf{c}_{i}$ depends in part on $\varepsilon_{i}$ : the admission of patient $i$ to hospital $j$ takes into account information that is correlated with $\varepsilon_{i}$. The conventional probit model is then misspecified.

To develop a more plausible model of hospital choice, we assume that patients become infected with one of the many bacterial or viral agents that can cause pneumonia and it has been determined that they are sufficiently ill to benefit from inpatient treatment. At that point the patient (or the patient's agent) selects from the set of $J$ hospitals the hospital to which the patient will be admitted. The actual choice decision will be a complex function of many factors, such as severity of illness, characteristics of the hospital, the patient's primary care physician, etc. One important observable influence on choice is distance: previous research has shown that the farther a patient is from a hospital, the less likely is the patient to be admitted to that hospital, other observables constant. ${ }^{11}$

To present the reduced form model of hospital choice define the $(J-1) \times q$ matrix $\mathbf{Z}_{i}$, $\mathbf{Z}_{i}^{\prime}=\left[\mathbf{z}_{i 1}-\mathbf{z}_{i J}, \mathbf{z}_{i 2}-\mathbf{z}_{i J}, \ldots \mathbf{z}_{i, J-1}-\mathbf{z}_{i J}\right]$. Let the $(J-1) \times 1$ vectors $\eta_{i} \sim \mathrm{N}(\mathbf{0}, \Sigma)(i=1, \ldots, n)$ be

\footnotetext{
${ }^{11}$ See Luft et al. (1990) and Burns and Wholey (1992).
} 
mutually independent conditional on the exogenous variables; let $\Sigma=\left\lfloor\sigma_{i j}\right\rfloor$. The hospital choice multinomial probit $\mathbf{c}_{i}^{*}=\left(c_{i 1}^{*}, \ldots, c_{i, J-1}^{*}\right)^{\prime}$ is a $(J-1) \times 1$ latent vector,

$$
\mathbf{c}_{i}^{*}=\mathbf{Z}_{i} \alpha+\eta_{i}
$$

and $c_{i J}^{*}=0$. The choice indicator vector $\mathbf{c}_{i}=\left(c_{i 1}, \ldots, c_{i J}\right)^{\prime}$ has entry $c_{i j}=1$ if $c_{i j}^{*} \geq c_{i k}^{*}(k=1, \ldots, J)$ and $c_{i j}=0$ otherwise. Note that scaling $\alpha$ by any positive constant and $\Sigma$ by the square of that constant leaves the distribution of $\mathbf{c}_{i}$ conditional on $\mathbf{Z}_{i}$ unaffected. This identification problem is addressed subsequently in Section 2.2.

To permit unobserved severity of illness to affect hospital choice in any way consistent with this specification, the only restriction we place on the $(J-1) \times 1$ vector $\pi$ in

$$
\operatorname{var}\left(\varepsilon_{i}, \eta_{i}^{\prime}\right)=\left[\begin{array}{cc}
\sigma^{2} & \pi^{\prime} \\
\pi & \Sigma
\end{array}\right]
$$

is that it be consistent with $\operatorname{var}\left(\varepsilon_{i}, \eta_{i}^{\prime}\right)$ being positive definite. Since this implies complicated restrictions on $\pi$, a more graceful treatment is to work with the population regression of the shock $\varepsilon_{i}$ in (1) on the shock vector $\eta_{i}$ in (2),

$$
\varepsilon_{i}=\eta_{i}^{\prime} \delta+\zeta_{i} ; \operatorname{cov}\left(\eta_{i}, \zeta_{i}\right)=0
$$

In this regression $\delta \in \mathfrak{R}^{J-1}$ and the scale is normalized by $\operatorname{var}\left(\zeta_{i}\right)=1$. This specification simultaneously resolves the identification problem due to the scaling in (2) and incorporates all permissible values of $\pi=\Sigma \delta$ in (3). The variance of the shock in the mortality probit equation is $\sigma^{2}=\delta^{\prime} \Sigma \delta+1$, and the correlation between $\varepsilon_{i}$ and $\eta_{i j}$ is $\rho_{j} \equiv\left(\sum_{k=1}^{J-1} \delta_{k} \sigma_{k j}\right) /\left[\sigma_{j j}(\delta \Sigma \delta+1)\right]^{\prime 2}$. In the hypothetical experiment in which patient $i$ is admitted to hospital $j$ by means of a random assignment $\mathbf{c}_{i}, \mathrm{P}\left(m_{i}=1 \mid \mathbf{x}_{i}\right)=\Phi\left[\left(\mathbf{w}_{j}^{\prime} \beta+\mathbf{x}_{i}^{\prime} \gamma\right) /(\delta \Sigma \delta+1)^{1 / 2}\right]$.

We shall refer to $q_{j} \equiv-\mathbf{w}_{j}^{\prime} \beta /(\delta \Sigma \delta+1)^{1 / 2}$ as the hospital $j$ quality probit. Differences in these probits across hospitals may be used to address quality comparisons for individual hospitals. To compare groups of hospitals, we shall make use of the quantities $q_{G}=\sum_{j \in G} \omega_{j} q_{j}$, where $\mathrm{G}$ is the group of interest and $\left\{\omega_{j}\right\}$ is an appropriate set of weights. In the conventional probit model with normalization $\sigma=1$, the hospital $j$ quality probit is $q_{j}^{*}=-\mathbf{w}_{j}^{\prime} \beta$. We shall refer 
to $\rho_{j}$ as the hospital $j$ severity correlation. These correlations subsequently play an important role in explaining differences between $q_{j}$ and $q_{j}^{*}$.

\subsection{Prior distributions}

Given the complexity of the model and the low signal-to-noise ratio in the data, the prior distribution must be chosen carefully to reflect reasonable beliefs about hospital choice and mortality. The number of parameters in the variance matrix $\Sigma$ in the reduced form multinomial probit model for hospital choice is $J(J-1) / 2$, that is, 6,786 in our sample with $J=117$ hospitals. Identifying scale normalization reduces the number by only one. Because of the large number of parameters, and because the purpose of this study is to model mortality while permitting non-random hospital admission rather than to study the hospital admission process per se, we fix $\Sigma=\mathbf{I}_{J-1}+\mathbf{e}_{J-1} \mathbf{e}_{J-1}^{\prime}\left(\mathbf{e}_{n}\right.$ denoting an $n \times 1$ vector of units). This is the variance matrix that would result if the random components of utility associated with hospital admission were independently and identically distributed across hospitals prior to normalization on the utility of the last hospital choice by differencing. The prior distribution of $\alpha$, the coefficient

vector in the multinomial probit model, is $\mathrm{N}\left(\mathbf{0}, .5 \mathbf{I}_{q}\right)$. Distance is measured in hundreds of kilometers, so that Los Angeles County is (very roughly) one unit square. The other four variables in the choice equation are normalized similarly. Thus the prior is neutral about the relation between distance of the patient from the hospital and hospital choice, but at the same time permits the probability of admission to a hospital on the other side of Los Angeles County to be very small relative to the probability of admission to a hospital in the patient's immediate neighborhood. In fact, the signal-to-noise ratio for the choice equation is high, because there are only five free parameters. The data turn out to be much more informative than the prior for these parameters.

The prior distribution for $\delta$, the coefficient vector in the population regression (4) of the mortality shock on hospital choice shocks, is $\delta \sim N\left(\mathbf{0}, .5 \mathbf{I}_{J-1}\right)$. Since the variance of the mortality shock is $\delta^{\prime} \Sigma \delta+1$, the prior mean of this variance is J. The squared correlation between the mortality shock and a hospital choice shock $\eta_{i j}$ is

$$
\rho_{j}^{2}=\left(\sum_{k=1}^{J-1} \delta_{k} \sigma_{k j}\right)^{2} / \sigma_{j j}(\delta \Sigma \delta+1)=\left(\delta_{j}+\sum_{k=1}^{J=1} \delta_{k}\right)^{2} / 2(\delta \Sigma \delta+1) .
$$


At the prior mean $\delta_{k}=0(k=1, \ldots, J)$, the correlation is 0 . On the other hand, the expectation of the numerator in (5) is $.5 \mathrm{~J}$ and that of the denominator is $2 \mathrm{~J}$, so correlation coefficients of 0.5 are reasonable under this prior also. Thus the implied prior on $\pi$ in (3) is not very strong.

The coefficients $\beta_{j}$ in the mortality probit equation are independent in the prior. For the intercept, $\beta_{1} \sim \mathrm{N}(-4,2)$. For the coefficients corresponding to size and ownership categories, $\beta_{i} \sim \mathrm{N}(0,2)(i=2, \ldots, 9)$. For the hospital specific coefficients, $\beta_{i} \sim \mathrm{N}(0, .5)(i=10, \ldots, J+9)$. These prior distributions resolve the perfect multicollinearity problem in the matrix of indicators $\mathbf{W}$, in the sense that the posterior distribution is proper. ${ }^{12}$ Since any hospital belongs to exactly four classes (the universal class, one size class, one ownership class, and the class unique to the hospital) the prior distribution of each element of any vector $\mathbf{w}_{j}^{\prime} \beta$ is $\mathrm{N}(-4,6.5)$. The prior places non-negligible support on values of $q_{j}=\mathbf{w}_{j}^{\prime} \beta /(\delta \Sigma \delta+1)^{1 / 2}$ between $(4-2 \sqrt{6.5}) / \sqrt{J}$ and $(4+2 \sqrt{6.5}) / \sqrt{J}$, i.e. the interval $(-0.1,9.1)$, since $J=117$. Roughly speaking, hospital quality probits implying that a randomly selected patient would have a mortality probability between 0 and .5 are plausible in the prior. In the sample, mortality frequency ranges 0.024 and 0.224 across all 117 hospitals. Thus the prior is uninformative relative to the data regarding the overall mortality rate.

This prior provides more structure on quality comparisons between hospitals. If hospitals $j$ and $k$ are in the same size and ownership classes then $\mathbf{w}_{j}^{\prime} \beta-\mathbf{w}_{k}^{\prime} \beta \sim \mathrm{N}(0,1)$; if they share one class in common then $\mathbf{w}_{j}^{\prime} \beta-\mathbf{w}_{k}^{\prime} \beta \sim \mathrm{N}(0,5)$; and if they share no classes in common $\mathbf{w}_{j}^{\prime} \beta-\mathbf{w}_{k}^{\prime} \beta \sim \mathrm{N}(0,9)$. Keeping in mind that the variance of the mortality probit shock is $\delta \Sigma \delta+1$ and recalling that the overall mortality rate for the sample is about .10, the prior expresses the belief that for hospitals in the same size and ownership classes mortality rates in the conceptual random assignment experiment should not differ by more than a percentage point or two. At the same time, differences across size and ownership categories can be much greater. The prior takes essentially no stance on the actual mortality rate for any given hospital, or on whether rates are higher or lower for particular classes.

\footnotetext{
${ }^{12}$ Of course, since it is only the prior distribution that distinguishes among the $\beta_{i}$, the posterior variance of no $\beta_{i}$ will approach 0 as sample size increases. However for any given hospital $j$ the posterior variance of $\mathbf{w}_{j}^{\prime} \beta$ will enjoy this feature (under obvious regularity conditions) and this is the feature that matters for the conceptual random assignment experiment that motivates this study.
} 


\subsection{Inference}

The observed data are $\mathbf{W}$ and $\left(\mathbf{x}_{i}, \mathbf{Z}_{i}, \mathbf{c}_{i}, \mathbf{m}_{i}, i=1, \ldots, n\right)$, which can be abbreviated as $\mathbf{y}$. The model contains latent variables $\left(m_{i}^{*}, \mathbf{c}_{i}^{*}, i=1, \ldots, n\right)$, which can be abbreviated $\mathbf{y}^{*}$. The parameter vectors are $\alpha, \beta, \gamma$ and $\delta$, which can be collected in the vector $\theta$. The model specified in Section 2.1 provides $\mathrm{p}\left(\mathbf{y}, \mathbf{y}^{*} \mid \theta\right)$ and the prior distributions in Section 2.2 provide $\mathrm{p}(\theta)$. Explicit expressions for these densities are given in the appendix. From Bayes rule, the distribution of the unobservables $\mathbf{y}^{*}$ and $\theta$ conditional on the data and model specification is

$$
\mathrm{p}\left(\mathbf{y}^{*}, \theta \mid \mathbf{y}\right)=\frac{\mathrm{p}(\theta) \mathrm{p}\left(\mathbf{y}, \mathbf{y}^{*} \mid \theta\right)}{\mathrm{p}(\mathbf{y})} \propto \mathrm{p}(\theta) \mathrm{p}\left(\mathbf{y}, \mathbf{y}^{*} \mid \theta\right) .
$$

The objective is to obtain the posterior distribution of functions like the hospital quality probits $q_{j}$, and $\Phi\left[\left(\mathbf{w}_{j}^{\prime} \beta+\mathbf{x}_{i}^{\prime} \gamma\right) /(\delta \Sigma \delta+1)^{1 / 2}\right]$, the probability of mortality under random hospital admission of a patient with observed characteristics $\mathbf{x}_{i}$ to hospital $j$. A closely related quantity of interest is $\mathrm{P}\left(q_{j}>q_{k} \mid \mathbf{y}\right)$, the posterior probability that hospital $j$ ranks above hospital $k$ in the conceptual motivating experiment. This objective requires integrating a highly nonlinear function over millions of dimensions, most of which correspond to latent variables. It cannot be achieved through analytical means.

Instead, we take advantage of the fact that the parameter vector and latent variables can be partitioned into groups, such that the distribution of any one group conditional on all the others is of a single, easily recognized form that is easy to simulate. Details of the partition are given in the appendix. The problem is thus well suited to attack by execution of a Gibbs sampling algorithm (Gelfand and Smith, 1990; Geweke, 1999). In this approach, each group of parameters and latent variables is simulated conditional on all the others. Following each pass through the entire vector of latent variables and parameters, all parameter values are recorded in a file.

As detailed in the appendix, the Gibbs sampling algorithm is ergodic and its unique limiting distribution is the posterior distribution. Therefore, dependent draws from the posterior distribution of any function of the parameters $\mathrm{g}(\theta)$ can be made by computing the value of $\mathrm{g}$ corresponding to the recorded parameter values, after discarding initial iterations of the Gibbs sampling algorithm to allow for convergence. We used parallel computing methods and a 
supercomputer, exploiting the fact that in each iteration of the Gibbs sampling algorithm the latent variables $\left(m_{i}^{*}, \mathbf{c}_{i}^{*}, i=1, \ldots, n\right)$ are conditionally independent across individuals. The iterations themselves are executed serially. The results reported in Section 4 are based on 38,000 successive iterations, after discarding 15,000 iterations based on convergence diagnostics. For comparison purposes, the same procedures were applied to a conventional probit model for mortality, with the variance of $\varepsilon_{i}$ in (1) fixed at $\sigma=1.0$. This computation, which is much simpler, is based on 35,000 iterations of the Gibbs sampling algorithm described in Albert and Chib (1993).

\section{The Data}

The primary source of data for this study is the Version B Discharge Data from the State of California Office of Statewide Health Planning and Development. These data provide records for all patients discharged from any California acute-care hospital during the years 1989 through 1992. We chose to analyze four years because the number of patients per hospital was then large enough to obtain meaningful inference but small enough to be computable. We did not choose more recent data, because increased managed care penetration among Medicare enrollees during the 1990s would add complicating factors to the choice data. We confine our attention to those patients admitted to a hospital in Los Angeles County. A large metropolitan area is best suited to our purposes, because it has a large base of patients and contains multiple hospitals in every size and ownership class. We limit our study to a single disease, because there is evidence that severity mechanisms work best when they are disease specific. ${ }^{13}$ We choose pneumonia in particular for three reasons. First, it is a common disease ${ }^{14}$ that provides the large sample needed to draw inferences about hospital quality. Second, in-hospital death is a relatively frequent outcome for pneumonia patients, which makes it an attractive disease to examine through the medium of hospital discharge records. Third, there is independent evidence that an appropriately adjusted in-hospital mortality rate for pneumonia is correlated with the quality of in-hospital

\footnotetext{
${ }^{13}$ See Wray et al. (1997)

${ }^{14}$ Pneumonia and influenza alone constitute the sixth leading cause of death in the US, and the fourth leading cause of death for those over 65 (National Center for Health Statistics, 1996). Pneumonia is also the leading cause of death among patients with nosocomial (hospital acquired) infections (Pennington, 1994).
} 
care. ${ }^{15}$ We further confine our attention to patients who were over 65 at the time of admission. Medicare is the common primary source of medical insurance for this group, and in the case of pneumonia limiting the study to patients over 65 leaves a large patient base.

The secondary source of data is the Annual Survey of Hospitals Database published by the American Hospital Association (AHA). Among other information, the AHA data contain the addresses, ownership status, and size of each hospital in our sample.

\subsection{Sample construction}

The sample was selected through a process of eliminating patients from the 1989-1992 Version B discharge Data. The first qualification for selection is that the patient be admitted to a Los Angeles County hospital and over 65 at the time of admission.

The second qualification is that one of the five ICD-9-CM disease codes specified in the discharge data be 48.1, 48.2, 48.5, 48.6, or 48.38. This procedure is suggested by Iezzoni (1996) to define pneumonia. There is substantial non-random variation across hospitals in the sequence of ICD-9 diagnoses listed. Thus, choosing the first listed ICD-9 code may induce biases. (Iezzoni (1997), Chapter 3).

The third qualification is that the source of admission must be either routine, or from the emergency room. This eliminates patients transferred into the hospital from another medical facility, or admitted from an intermediate care or skilled nursing facility. To the extent that placement in these facilities is correlated with unobserved disease severity, and to the extent that such facilities may be systematically located near higher quality hospitals, the key assumption that distance from the hospital is exogenous in our model would be violated. This step eliminates approximately 23 percent of the patients from the sample.

The fourth qualification for inclusion in the sample is that the patient be admitted to a hospital with at least 80 admissions for pneumonia in our data set. This qualification was imposed for two reasons. First, the fewer admissions to a hospital in our data the less we can learn about the quality of that hospital. The hospitals eliminated through this qualification would have had a very low signal-to-noise ratio. Second, computation time in the Gibbs sampling algorithm is largely driven by the number of latent variables. To have included the 14 hospitals eliminated through this qualification would have markedly increased computation costs while

\footnotetext{
${ }^{15}$ See Keeler et al. (1990) and McGarvey and Harper (1993).
} 
providing little additional information about the unknown parameters. In principle, this qualification introduces a problem of choice based sampling, but because only 431 patients were thereby eliminated we believe that this is a negligible difficulty.

\subsection{Variable construction}

The covariate vector $\mathbf{x}_{i}$ in the mortality probit equations contains demographic variables and indicators of disease severity. Most of the demographic variables are constructed from the discharge records. These are four age indicators (70-74, 75-79, 80-84, and 85 or older), an indicator for female, and indicators for black, Hispanic, Native American and Asian respectively. The discharge records contain no information on socioeconomic status. As a proxy for the patient's household income, we use the mean 1990 census household income for householders with the same zip code, race, and age class as the patient. ${ }^{16}$

Indicators of disease severity in $\mathbf{x}_{i}$ are constructed from the admission disease staging information contained in the discharge records. Disease staging has been shown to be as good as some risk adjustment data based on chart review of medical records. ${ }^{17}$ Since some of the 13 stages have very few patients, we aggregated stages into five groups: stage 1.1, stages 1.3 through 2.3, stages 3.1 through 3.6, stage 3.7, and stage 3.8. Indicator variables for all but stage 1.1 are included in $\mathbf{x}_{i}$.

The indicator for mortality, $m_{i}$, is set to 1 if the patient died in the hospital within ten days of admission to the hospital; otherwise it is set to 0 . The horizon for mortality is limited to ten days, because beyond this point hospitals sometimes transfer terminally ill patients to other facilities, and this decision appears to vary considerably by hospital. To control for differential patient transfer, Gowrisankaran and Town (1999) used a hazard model as an alternative to the 10-day inpatient mortality, but found little difference between the two specifications. In two separate studies of heart disease patients, McClellan, McNeil and Newhouse (1994) and

\footnotetext{
${ }^{16}$ The census provides only two relevant age categories, $65-74$ and 75+, instead of four. Thus, we aggregated the discharge data age categories to this level. Additionally, the census provides income only within cells. To find the mean income, we took the mean value for each cell as the income for each household in that cell. For the highest cell, $\$ 100,000$ or more, we assumed a mean income of $\$ 140,000$.

${ }^{17}$ See Thomas and Ashcroft (1991). Iezzoni et al. (1996) showed excellent agreement of disease stage with the ratings of other systems.
} 
McClellan and Staiger (1999b), find that there is a very strong correlation between 7-day mortality and 30-day mortality. ${ }^{18}$

Table 1 provides a summary of the distribution of demographic characteristics and disease severity in the sample, together with mortality rates. For each age group the breakdown of the sample by race and sex closely reflects the demographics of Los Angeles County. Older individuals enter the sample in greater proportion to their numbers in the population than do younger ones. In each age group three-quarters of the sample is classified in the least severe disease stage. Mortality rates increase gradually with age, increase sharply with disease stage, are about the same for each sex, and are lower for Asians and Hispanics than for whites or blacks.

The covariate matrix $\mathbf{Z}_{i}$ contains variables specific to the combination of patient $i$ and each hospital. The additional information in $\mathbf{Z}_{i}$ not contained in $\mathbf{x}_{i}$ is the distance of the patient's home from each hospital. We obtained patient zip codes from the discharge data and the hospital zip codes from the AHA data. We then used the Census TIGER database to find the latitude and longitude of the centroid of each zip code. Given the latitudes and longitudes, we computed the distance between each patient home and hospital using standard great circle trigonometric formulas. ${ }^{19}$ We then constructed the five variables in $\mathbf{Z}_{i}$ : distance (always measured in hundreds of kilometers); distance-squared; the product of distance and an age indicator (1 for 65-69, 2 for 70-74, 3 for 75-79, 4 for 80-84, 5 for 85+); the product of distance and disease stage (1.1, 1.2, $\ldots, 3.8$ ); and the product of distance and income (in units of $\$ 100,000$ ).

The hospital characteristic matrix $\mathbf{W}$ contains indicator variables described in detail in Section 2.1. This information was obtained from the AHA survey, and is summarized in Table 2. Note that we have grouped private teaching hospitals as a separate ownership category from private not-for-profit hospitals. Most hospitals are private, split about evenly between for-profit and not-for-profit. Only nine of the 117 hospitals in the sample are teaching or public, but on average they are larger than private hospitals and together admitted almost $12 \%$ of the patients in the sample. Slightly less than one-quarter of the hospitals are classified in the largest size group (at least 300 beds) but they account for over $40 \%$ of the admissions in our sample.

\footnotetext{
${ }^{18}$ As caveats, note that heart disease is very different from pneumonia and that these studies examine mortality, not inpatient mortality.

${ }^{19}$ For zip codes that contain more than one hospital, we used address-level latitude and longitude data from the Census TIGER database, which stores the geographic location of every block corner and will interpolate from that to find the latitude and longitude of any address.
} 
Mortality rates differ only slightly by ownership category, with the lowest rates in teaching hospitals and the highest in private not-for-profit hospitals. Under the naïve interpretation of the data as having arisen from a randomized experiment, there is little evidence that mortality rates are higher in any one ownership category than any other. The most informative comparison is of private not-for-profit hospitals with teaching hospitals: beginning with independent flat priors on mortality probabilities, the posterior probability that the private not-for-profit mortality rate is lower than the teaching mortality rate is .07. Similar comparisons for other ownership categories yield posterior probabilities between .10 and .09 . There is greater variation in mortality rates by hospital size, with the small hospitals having lower rates than the other three categories. Beginning with the same flat prior, the posterior probability that this is true of population mortality rates is greater than .950 for all three comparisons. However other such comparisons across size categories yield posterior probabilities between .15 and .85 .

Comparisons of mortality rates between cross-classified cells yields are more complex. The four private not-for-profit hospitals in the 150-200 bed category have the highest mortality rate, exceeding that of smaller hospitals in the same ownership category (posterior probability .995), hospitals with 200-299 beds in the same ownership category (posterior probability .992) and that of private for-profit hospitals of the same size (posterior probability .984). The single large private for-profit hospital has a mortality rate that exceeds that of large hospitals in all other ownership categories, though only the comparison with teaching hospitals yields a posterior probability above .90 . Its rate also exceeds that of all other size categories for private for-profit hospitals, the comparison with the smallest size category yielding a posterior probability above .90 .

Table 3 summarizes the distribution of severity of illness, as measured by disease stage, across the different categories of hospitals. Patients in larger hospitals tend to be at a more advanced disease stage. The differences in the distribution are small, but because of the large sample size they are highly significant: the test statistic for categorical independence is $\chi^{2}(12)=63.1\left(p=6.11 \times 10^{-9}\right)$. The distribution of patients by disease stage over hospitals of different ownership type is yet more uneven: almost $79 \%$ of the patients in teaching hospitals are in the earliest disease stage, whereas at private for-profit hospitals only a little over $74 \%$ are at this stage. The test statistic for categorical independence is $\chi^{2}(12)=179.0\left(p=6.84 \times 10^{-32}\right)$. Thus it is the case in this data set, as in similar data sets, that observed severity is not randomly 
distributed across hospitals. This underscores the importance of examining and controlling for nonrandom assignment by unobserved severity, as well.

The summaries of the data provide no simple interpretation of mortality rates. They indicate systematic differences in measured disease severity across hospitals by size and ownership classes. They hint at the possibility of important differences between individual hospitals within size and ownership classes. Thus we now turn to the application of the model developed in Section 2 to inform our understanding of the relationship between choice of hospital admission and mortality.

\section{Findings}

The model set forth in Section 2 applied to the data described in Section 3 yields evidence on systematic differences in quality across hospitals, provides insight into the interaction between hospital choice and hospital quality, and suggests quality orderings among hospitals. This section summarizes these findings, using the selection model and the probit model. The common center of the two models is the mortality probit equation

$$
m_{i}^{*}=\mathbf{c}_{i}^{\prime} \mathbf{W} \beta+\mathbf{x}_{i}^{\prime} \gamma+\varepsilon_{i}
$$

discussed in Section 2.1. To recapitulate, each row $\mathbf{w}_{j}^{\prime}$ of $\mathbf{W}$ corresponds to a particular hospital, with $\mathbf{w}_{j}$ containing size and ownership category indicators for hospital $j$ as well as an indicator specific to hospital $j$. The vector $\mathbf{c}_{i}$ has a single non-zero element which indicates the hospital to which patient $i$ is admitted.

The probit model consists of equation (6), the specification $\varepsilon_{i} \sim \mathrm{IID}(0,1)$, and the assumption that the assignment process that generates $\mathbf{c}_{i}$ is independent of unobserved individual specific influences on mortality $\varepsilon_{i}$. The hospital $j$ quality probit in the probit model is denoted

$$
q_{j}^{*}=-\mathbf{w}_{j}^{\prime} \beta .
$$

The methods discussed in Section 2.3 provide a sample from the joint posterior distribution of all the hospital quality probits $q_{j}^{*}$.

The selection model adds to (6) the multinomial model for choice

$$
\mathbf{c}_{i}^{*}=\mathbf{Z}_{i} \alpha+\eta_{i} ; \eta_{i} \stackrel{I I D}{\sim} \mathrm{N}(\mathbf{0}, \Sigma)
$$


fully discussed in Section 2.1. In (8) $\mathbf{c}_{i}^{*}$ is a 117-element vector of choice utilities. The hospital chosen is the one with the highest value of $c_{i j}^{*}$. The shocks $\varepsilon_{i}$ and $\eta_{i}$ are jointly normally distributed. The interaction between $\eta_{i}$ and $\varepsilon_{i}$ is unrestricted and is indicated by the population regression of $\varepsilon_{i}$ on $\eta_{i}$,

$$
\varepsilon_{i}=\eta_{i}^{\prime} \delta+\zeta_{i} ; \operatorname{cov}\left(\eta_{i}, \zeta_{i}\right)=0 ; \zeta_{i} \sim \mathrm{IID} N(0,1)
$$

The variance of the shock to the mortality probit in (6) is therefore $\delta \Sigma \delta+1$ in the selection model. From (9), the hospital $j$ severity correlation is

$$
\rho_{j}=\operatorname{corr}\left(\varepsilon_{i}, \eta_{i j}\right)=\left(\sum_{k=1}^{J-1} \delta_{k} \sigma_{k j}\right) /\left[\sigma_{i j}\left(\delta^{\prime} \Sigma \delta+1\right)\right]^{12}
$$

Because the selection model permits correlation between $\eta_{i}$ and $\varepsilon_{i}$, the posterior distribution for $\beta$ and $\gamma$ in this model is not the same as it is in the probit model. Inferences about quality therefore differ as well. To emphasize this fact while permitting comparisons between the two models, the hospital quality probit is defined

$$
q_{j}=-\mathbf{w}_{j}^{\prime} \beta /\left(\delta^{\prime} \Sigma \delta+1\right)^{V_{2}}
$$

in the selection model.

In studying systematic differences in hospitals it is useful to use weighted averages of the quality probits $q_{j}$ or $q_{j}^{*}$ across certain hospitals to form group hospital quality probits $q_{G}$ or $q_{G}^{*}$ in the respective models. The weights used are the number of patients admitted to the hospitals. These are the same weights used to summarize the data in Section 3.

\subsection{Parameter estimates}

Table 4 presents the posterior means and standard deviations of some parameters and functions of parameters in the selection and probit models. In the case of the selection model Table 4 presents the posterior means and standard deviations of $\gamma_{j} /(\delta \Sigma \delta+1)^{1 / 2}$ or of the negative of the group hospital quality probit $-q_{G}$. The normalization facilitates comparison between models and interpretation of the functions of interest as probits.

The mortality equation has three groups of covariates: demographics, disease severity, and hospital indicators. In the case of the demographic and disease severity covariates, coefficient posterior means in the selection and probit models are similar to each other, and closely reflect the mortality rates presented in Table 1. Posterior standard deviations indicate substantial 
information about differences in mortality probabilities across demographic groups. This, too, is not surprising in view of the summary statistics in Table 1.

In the case of the hospital quality probits, there are greater and more interesting differences between the selection model, the probit model, and Table 2. In all three approaches the smallest hospitals have lower mortality rates than larger hospitals. The hospital mortality rate is highest for the largest hospitals in the raw data, whereas it is for 200-299 bed hospitals in the selection model and for 150-199 bed hospitals in the probit model. The selection model finds more systematic variation in quality by size, than does the probit model. Table 5 provides explicit posterior probabilities for hospital group quality comparisons. In the selection model, the posterior probability that the group hospital quality probit for the smallest-hospital group exceeds that of the largest-hospital group is 0.889 , and the posterior probability that it exceeds that of the other two size groups exceeds 0.99 . The posterior probability that the group hospital quality probit for the largest-hospital group exceeds that of the 150-199-bed group is 0.956, and the posterior probability that it exceeds the 200-299-bed group is 0.991. These distinctions are both different and sharper than those using the raw data (Table 1) which does not control for demographic characteristics and observed disease severity, and implicitly assumes a random assignment of patients to hospitals.

We know of only one other study that has specifically studied the relationship between hospital quality and size for pneumonia. This study, Gowrisankaran and Town (1999), found that the relationship between quality and size varies by for-profit status. Another related study is a study by Keeler et al. (1992), that examined the relationship between hospital quality and size using a very detailed and expensive data set that included pneumonia patients along with patients with other, more complex diagnoses. They found that hospital quality increases with bed size. The difference between our results and theirs may be due to the nature of the treatment of pneumonia versus more complex procedures. Successful pneumonia treatments are linked to identifying the pathogen responsible for the infection and administering the appropriate antibacterial agent early in the progression of the disease, and subsequently monitoring and adjusting the dosage of the drug (Rello and Valles (1998), Pennington (1994), McGarvey and Harper (1993)). There is evidence that smaller hospitals may be better at the timely administration of antibiotics (Fine et al. (1998)) which may explain why we observe that they have better outcomes. Furthermore, since small hospitals are likely to treat a disproportionate 
number of pneumonia patients relative to more technically challenging illnesses ${ }^{20}$ they may also develop expertise in this disease. That, in turn, may overcome advantages that large hospitals may have in other dimensions, such as laboratory facilities.

Contrasts between the respective models and the raw data are even greater for classification by ownership. The probit model does not distinguish between any of the four types: differences are small and insignificant. The selection model draws a sharp distinction between private hospitals: for-profits have a markedly higher quality probit than not-for-profits, and the posterior probability of this ordering is 0.999 . In the raw data teaching hospitals show significantly lower mortality rates than the other ownership categories. In the selection model the sharpest distinction that can be drawn for teaching hospitals is that the posterior probability that the quality probit for this group is lower than that of for-profit private hospitals is 0.95 .

There is debate in health policy circles regarding the role that for-profit hospitals should play in the U.S. health system (Gray (1991)). Some have argued that private, not-for-profit hospitals may better serve the public interest because they are more likely to provide better care. Our results unequivocally indicate that for the treatment of pneumonia in older patients and the hospitals in our sample, the opposite is true. Private, for-profit hospitals provide better care than private not-for-profit or public hospitals for pneumonia. Keeler et al. (1992) also found that public hospitals in large cities to be of lower quality, while the difference in quality between forprofit and not-for-profit hospitals is less pronounced. McClellan and Staiger (1999a) conclude that the quality difference in for-profit and not-for-profit hospitals is small and if anything forprofits likely provide better care in the treatment of heart attacks. Teaching hospitals, which are generally viewed as providing superior care, do not offer significantly higher quality according to the selection model. Consistent with our finding, Fine et al. (1998) found that major teaching hospitals were less likely to be timely with administration of antibiotics than non-teaching hospitals.

The advantage of the probit as the unit of comparison is that it provides differences in mortality probability beginning from any base level probability: probability differences are greatest when the base probability is 0.5 , and decline monotonically for lower and higher

\footnotetext{
${ }^{20}$ Performing a simple multinomial logit regression of Southern California patients, we found that pneumonia patients were more likely to be admitted to smaller hospitals than were the average hospital patient. In contrast, acute myocardial infarction (heart attack) patients were more likely to be admitted to larger hospitals than the average hospital patient. Unlike pneumonia treatments, acute myocardial infarction treatments often include hightechnology surgery such as cardiac catheterization, angioplasty or bypass.
} 
probabilities. The disadvantage is that the implied probabilities are immediately clear only to the reader experienced in using probits. Table 6 conveys the comparison information, beginning from a base mortality probability of 0.10 , which is typical for pneumonia in admitted patients over the age of 65 . In the conceptual experiment underlying these tables, a patient is assumed to be admitted to one type of hospital with a 0.10 mortality probability, and the mortality probability for that patient in other types of hospitals is then inferred from the data and the model. The cell entries are posterior means of the latter probabilities, and the corresponding posterior standard deviations are indicated parenthetically. For changes in hospital size (Panel A), the range of probability differences is greatest in the comparison of the smallest hospitals with 200-299-bed hospitals, where the posterior distribution indicates an increase of $20 \%$ in moving from the former to the latter. Other changes are somewhat smaller, but the direction of change is often clearly indicated by the data and the selection model. In the case of classification by ownership (Panel B), changes in probability are similar. Consistent with Table 4, contrasts are most marked in movements to or from private for-profit hospitals. The largest posterior mean is an increase in mortality probability of $25 \%$ associated with a move from a private for-profit hospital to a public hospital. The model and data are most informative (in the sense that the direction of change is essentially certain) about a move from a private for-profit hospital to a private not-for-profit hospital.

The choice of hospital is governed by the multinomial probit model (6). Posterior means and standard deviations of the covariate coefficient vector $\alpha$ are presented in Table 7. As expected, distance is an important factor in describing the hospital of admission. To interpret the posterior mean of -7.17 , recall that the disturbances in (6) all have variance 2 , and that all disturbance covariances are 1 . The difference of any two disturbances also has variance 2 . Hence the posterior mean of -7.17 implies that a hospital that is 20 kilometers farther from a patient's home than another has a normalized probit that is $7.17 \times 0.2 / / \sqrt{2} \approx 1$ unit lower. The quadratic term in the equation is highly significant, but since distances are at most 100 kilometers within Los Angeles County, its substantive effect is not great.

Other covariates have important impacts on hospital choice as well. Interactions of distance with age, and with severity, have negative coefficients with small posterior standard deviations. Since the age variable is at least 1 and the severity variable is at least 1.1 , this lowers the posterior mean of the distance coefficient downward from -7.17 , at least as far as -8.59 for 
everyone in the sample, and to -13.55 for the oldest ( $85+$, coded 5) and most severely ill (disease stage 3.8) patients in the sample. Older and more severely ill patients clearly have the greatest propensity to be admitted to hospitals closer to where they live. The reason for this is likely due to the increased cost and difficulty of transport for severely ill patients. Patients from areas with higher income also tend to be admitted to hospitals closer to home, but the effect is not well determined and is small given the limited range of average income by zip code.

\subsection{Selection and selection bias}

A model that assumes random assignment of patients, like the simple probit model (1) taken alone, will provide mistaken inferences about hospital quality to the extent that conditional on covariates assignment is not independent of mortality. Our selection model provides the joint posterior distribution of hospital quality $q_{j}$ (defined in expression (11)) and hospital severity correlation $\rho_{j}$ (defined in expression (10)) across all 117 hospitals in the sample. The relation between $q_{j}$ and $\rho_{j}$ provides an interpretation of the role of unobserved severity in hospital admission, through the lens of our selection model. The relation between hospital quality $q_{j}^{*}$ (expression (7)) in the probit model, and $q_{j}$ and $\rho_{j}$ in the selection model, provides some insight into the nature of selection bias in the probit model as well.

The inferred relation between $q_{j}$ and $\rho_{j}$ in the selection model can be examined in several ways. Figure 1 provides a scatter plot of the posterior means of $q_{j}$ and $\rho_{j}$ for the 117 hospitals in the sample. This figure suggests a positive correlation between the posterior means, but not a strong relationship. This is borne out more formally in Table 8: the correlation between posterior means is 0.343 (Panel A) and a simple least squares regression of the posterior means of the $\rho_{j}$ on the posterior means of the $q_{j}$ shows a slope coefficient of 0.181 that is significantly positive $(t>4)$.

To interpret the relation between $q_{j}$ and $\rho_{j}$, consider a patient with high unobserved severity of illness and therefore a large value of $\varepsilon_{i}$ in the mortality equation (6). As $\varepsilon_{i}$ increases, this patient is increasingly likely to be admitted to hospitals with larger values of $\rho_{j}$ rather than hospitals with smaller values of $\rho_{j}$ because the shocks $\eta_{i j}$ in the choice model (8) are likely to be higher for these hospitals. Hospitals with high severity correlations $\rho_{j}$ also tend to be 
hospitals with higher quality $q_{j}$. Thus, other things equal, patients with greater unobserved severity of illness are more likely to be admitted to higher quality hospitals.

In contrast, the evidence on the correlation between hospital quality and observed risk factors is mixed. There is a small but positive correlation of 0.155 between the mean patient disease stage for a hospital and its mean posterior quality. Moreover, this correlation is significantly positive, when evaluated as a function of the posterior. However, the correlation between the mean observed patient severity of a hospital, $\bar{x}_{j} \gamma$, and the hospital quality $q_{j}$ is tiny $(-0.02)$ and insignificant. There are likely to be two motivating factors for this mixed result, both related to choice. First, there is some evidence that for-profit hospitals disproportionately locate in areas with high health status. ${ }^{21}$ In combination with the fact that for-profit hospitals are estimated to be of higher quality than average in our data, this suggests that high quality hospitals may locate in areas with low observed patient severity of illness, and particularly in areas with a low percentage of very old people. Second, from Table 7, we know that risk factors such as disease stage and age make a patient less likely to travel to obtain care. This is particularly true for age. Thus, patients who are very old may value closeness relatively highly and hence choose to seek care relatively close to home instead of seeking a high quality hospital. Nonetheless, for a given distance, they may be more likely to choose a high quality hospital.

It is clear from Figure 1 that many hospitals above average in quality have negative severity correlations, and likewise many hospitals of lesser quality have positive severity correlations. The first two columns of Table 9 provide more detail for seven hospitals in the sample. The first two are high in quality and severity correlation, the second two are low in both, and the last three are high in one and low in the other. Table 9 also indicates the substantial posterior uncertainty about hospital qualities and severity correlations that is not conveyed by the posterior means plotted in Figure 1. Hospital by hospital, there is substantial uncertainty about $q_{j}$ and $\rho_{j}$, but informative contrasts can still be drawn for many hospitals. For example, from the output of the posterior simulator the posterior probability that the quality of the first hospital is higher than that of the third is _. Contrasts among severity correlations are somewhat sharper. The posterior

\footnotetext{
${ }^{21}$ Norton and Staiger (1994) find location differences between for-profit hospitals and not-for-profit hospitals. In particular, for-profit hospitals locate in areas with few uninsured patients. These patients are likely to have low health status.
} 
probability that the severity correlation coefficient in the first hospital is higher than that in the third is $\ldots .{ }^{22}$

The impact of non-random assignment on inferred hospital quality arises hospital by hospital, and could be present even if there were no systematic relationship between qualities and severity correlations. In any selection model, for each hospital, conditional on observed characteristics (including observed severity) the observed mortality rate will be decomposed into a hospital quality component and an unobserved severity component. This is indicated clearly in the last column of Table 9, which provides the posterior means and standard deviations of the hospital quality probits $q_{j}^{*}$ in the simple probit model. Starting from quality in the selection model (first column) a high value of the severity correlation (second column) leads to a lower value of inferred quality in the probit model (third column). Conversely, a low value of the severity correlation-i.e., the hospital tends to get patients with low unobserved severity-raises inferred quality in the probit model relative to the selection model. The differences in $q_{j}$ and $q_{j}^{*}$ are striking. They plainly affect comparisons, a topic addressed in Section 4.3.

Panel $\mathrm{C}$ of Table 8 shows that this relationship is regular and not limited to the seven hospitals studied in Table 9. The posterior means for hospital quality $q_{j}^{*}$ in the probit model are well described as a linear function of hospital quality $q_{j}$ and severity correlation $\rho_{j}$ in the selection model. From the sample variation in $\rho_{j}$ apparent in Figure 1, and the regression relation reported in panel $\mathrm{C}$ of Table 8 , it is clear that variation in hospital severity correlation substantially drives variation in inferred hospital quality $q_{j}^{*}$ in the probit model. From the regressions in panels $\mathrm{B}$ and $\mathrm{C}$, one can infer the slope coefficient of .926 (= $1.404-2.639 x .181)$ in panel D. In the absence of information about $\rho_{j}$ (Panel D) there is no evidence against

\footnotetext{
${ }^{22}$ Since results in Table 7 are based on posterior means, they do not take into account dispersion in the posterior, either. Within the selection model this can be accommodated by regarding the sample relation between $q_{j}$ and $\rho_{j}$ as a function of the unknown parameters in the model, and then considering the posterior uncertainty associated with this relationship. To make this approach operational, one produces panels A and B of Table 7 for each draw from the posterior simulator, using the values of $q_{j}$ and $\rho_{j}$ for that simulation instead of the posterior means. This yields a posterior mean of _ _ and posterior standard deviation of _ for the sample correlation between $q_{j}$ and $\rho_{j}$ over the 117 hospitals. In the regression equation of panel B the posterior mean of the slope coefficient is _ and its posterior standard deviation is __. The OLS slope coefficient standard error has a posterior mean of __ and posterior standard deviation of __ ; " $t$ " has a posterior mean of __ and posterior standard deviation of __. Relationships among hospitals are thus not much affected by uncertainty about individual hospitals. As these results indicate, hospital-specific parameters are roughly independent in the posterior, and consequently relations between them are well summarized by relations between their posterior means.
} 
$\mathrm{E}\left(q_{j}^{*} \mid q_{j}\right)=q_{j}$ in a linear relation. But variation in hospital severity correlation accounts for a substantial portion of the variation in hospital mortality rates in the selection model, whereas in the simple probit model this variation must be attributed to quality differences. Consequently the probit model yields substantially greater variation in quality across hospitals than does the selection model. As suggested by the posterior standard deviations for the $q_{j}$ and $q_{j}^{*}$ in Table 9 , the probit model provides somewhat less information about $q_{j}^{*}$ than does the selection model about $q_{j}$. Over all 117 hospitals, the average posterior standard deviation for $q_{j}$ is .0879 in the selection model and for $q_{j}^{*}$ it is .0902 in the probit model.

\subsection{Ordering by quality}

The model and approach to inference described in Section 2 provide the complete posterior distribution of all the parameters in the model, and any functions of these parameters. In particular, corresponding to the parameter values in any iteration of the Gibbs sampling algorithm, it is a simple matter to compute the corresponding hospital quality probits $q_{j}$. The 38,000 successive iterations used to obtain the posterior moments reported in this section therefore also provide 38,000 draws from the joint distribution of the hospital quality probits $q_{j}$. Pairwise comparisons between hospitals are then straightforward. For example, for two hospitals $j$ and $k$, the numerical approximation to the posterior probability that $q_{j}>q_{k}$ is the fraction of iterations in which $q_{j}>q_{k}$, and the joint distribution of $q_{j}$ and $q_{k}$ could easily be plotted.

Comparing all 117 hospitals simultaneously is more challenging. A formal approach to ordering hospitals by quality would begin with a loss function for orderings. Suppose the 117element vector of quality ranks is $\mathbf{r}$, and the estimated quality rank vector is $\hat{\mathbf{r}}$. If the loss function is $(\hat{\mathbf{r}}-\mathbf{r})^{\prime} \mathbf{A}(\mathbf{r}-\hat{\mathbf{r}})$, where $\mathbf{A}$ is any positive definite matrix, then $\hat{\mathbf{r}}$ should be the posterior mean of $\mathbf{r}^{23}$ This estimate, may in turn be approximated numerically by sorting hospital qualities $q_{j}$ in each iteration of the Gibbs sampler, sorting ranks, and then averaging across all iterations. The resulting estimated ranks $\hat{r}_{j}$ are generally not integers.

\footnotetext{
${ }^{23}$ See, for example, Bernardo and Smith (1996, Section 5.1.5), for this standard result, as well as the one on medians used in the next paragraph.
} 
Table 10 provides the results of this approach for the selection model. The hospitals listed in the table are sorted by the values of the $\hat{r}_{j}$, and the number to the left of each hospital name indicates its order in this sorting. The $\hat{r}_{j}$ are shown in the second column following the hospital names. Posterior mean quality is shown in the first column following the names. Ordering by hospital quality posterior mean does not lead to the same ordering as ordering by $\hat{r}_{j}$, but it is very close. If the loss function were $\sum_{j=1}^{117} a_{j}\left|\hat{r}_{j}-r_{j}\right|$, where all $a_{j}>0$, then $\hat{r}_{j}$ should be the median of the posterior distribution of $r_{j}$, which in turn is an integer (with probability one). Medians are shown in the third column of Table 10. They provide yet another ordering, but it too is similar to ordering by mean quality and mean rank. We conclude that choice of loss function is not likely to affect orderings of point estimates of relative quality very much.

Of much greater significance is posterior uncertainty about comparative quality. Table 10 conveys this in several ways. The last four columns provide the probability of being in each quality quartile for each hospital. Placement within a quartile is most certain for hospitals of very high or very low quality. For those hospitals ordered 20 through 102 in Table 10, the posterior probability is at least .10 that the hospital is in one of each of three quartiles. The uncertainty conveyed by the posterior distribution is also reflected in the mean and median ranks. If there were no posterior uncertainty about ranks, the mean and median ranks would be identical to each other, and to the ordering number to the left of each hospital name. At the other extreme, if hospital qualities were completely exchangeable in the posterior distribution, the mean and median ranks would all be 59 . Note that the situation in Table 10 is intermediate between these two extremes, but closer to the former than the latter.

Table 11 provides an alternative expression of the uncertainty conveyed by the posterior distribution. Nine hospitals, including the first and last, were selected from roughly evenly spaced points in the ordering in Table 10. Then, pairwise posterior probabilities of orderings were computed from the iterations of the Gibbs sampler. For the first and last hospitals, fairly confident conclusions can be draw in comparisons with the other eight, but note that the posterior probability that the quality of Linda Vista exceeds St. Johns, and that Harbor/UCLA exceeds Verdugo Hills, are each above .10. For hospitals ordered 15, 30, 45, 60, 75, 90, and 105, only two orderings that can be made with .90 posterior probability: the quality of Linda Vista (ordered 15) exceeds that of Kaiser (ordered 75) and Verdugo Hills (ordered 105). Table 11 suggests that 
for half the hospitals in the sample (roughly those ordered 30 through 90 in Table 9) one cannot order quality pairwise with posterior probability that exceeds .80 .

The same set of comparisons using the probit model is made in Tables 12 and 13. These tables reflect the substantially different orderings, and greater range in quality, inferred using this model that assumes random assignment of patients to hospitals. For some hospitals the differences are enormous. To continue with two of the hospitals from Table 9, note that Pioneer Hospital is ordered $18^{\text {th }}$ by the selection model, but $109^{\text {th }}$ by the probit model. San Pedro Peninsula Hospital is ordered $72^{\text {nd }}$ in the selection model, but $19^{\text {th }}$ in the probit model. Correspondingly the selection model indicates a high hospital severity correlation in the former case and a low hospital severity correlation in the latter (Table 9).

While this study has presented arguments and evidence that the premise of conditionally random hospital admissions in the probit model is incorrect, it is interesting to compare the degree of confidence about orderings that this model yields with that in the selection model. To a rough approximation, the same difference between a hospital quality probit and the median quality probit leads to the same degree of confidence about rankings in each model. For example, the hospitals with posterior mean quality probit 0.1 below the median posterior mean quality, the posterior probability of being below the median is about .90 in each model, as read from the quartile probabilities in the last four columns. But because the qualities are more spread out in the probit model, sharper conclusions can be drawn about orderings. This overall pattern is evident in the pairwise comparisons in Tables 11 and 13. Section 4.2 argued that the additional dispersion in quality in the probit model arises precisely because selection is ignored. From this perspective, the greater posterior confidence that is seemingly inherent in the probit model is ironic.

Note that the information in Table 10 cannot be used to address the question of the range of quality over all 117 hospitals. The posterior mean of the highest quality probit of all hospitals exceeds that of the highest quality probit of any one hospital. This is reflected in the fact that the highest posterior mean rank in Table 9 is 4.5 , not 1.0. In the selection model highest quality has a posterior mean of .345 (posterior standard deviation .049), lowest quality has a posterior mean of -.365 (posterior standard deviation .061), and the range has posterior mean .711 (posterior standard deviation .082). There is considerable uncertainty about which hospitals have highest and lowest quality, and that is why the posterior mean range is considerably larger than the 
difference of .598 in posterior mean quality of St. Johns and Harbor/ULCA in Table 9. For the probit model the numbers are larger: highest quality .705 (.166); lowest quality -.786 (.183); and range $1.491(.215)$.

\section{Conclusion}

This study has extended existing econometric methods in order to measure hospital quality using the experience of patients admitted to hospitals in nonrandom fashion. Using discharge records for over 77,000 older pneumonia patients from 117 hospitals in Los Angeles County, we find strong evidence of differences in quality between hospitals of different size and ownership classifications. Smaller hospitals, and private for-profit hospitals, exhibit higher quality. We also detect substantial differences in quality for a sizable minority of individual hospitals.

As an important by-product, our methods produce information about the hospital admissions process. Patients with greater unobserved severity of illness tend, overall, to be admitted to hospitals of higher quality. Consequently more conventional methods that ignore nonrandom admission, when applied to this data set, tend to lower the inferred quality of good hospitals and raise that of poor ones, relative to our findings. We find that variation across individual hospitals in the unobserved severity of illness is at least as great as variation in quality, and that this

variation accounts for most of the large discrepancy between inference about hospital quality in our model and with more conventional methods.

The procedures used here are at the current frontier of intensive computational methods in econometrics. A supercomputer and several days of computing were required to obtain the results reported here. Recent and imminent innovations in numerical methods and computing technology should sharply reduce the real costs of these procedures in the near term. Given the policy importance of assessing quality of care in hospitals, we believe there is a significant return to further investment in these methods and their application to similar questions in health policy and related fields. 


\section{Table 1}

Frequency and Mortality Rates by Age, Disease Stage, Racial and Sex Categories

\begin{tabular}{|c|c|c|c|c|c|c|c|}
\hline \multirow{2}{*}{\multicolumn{2}{|c|}{$\begin{array}{c}\text { Severity and } \\
\text { Demographic } \\
\text { Categories }\end{array}$}} & \multicolumn{5}{|c|}{ Age Categories } & \multirow{3}{*}{$\begin{array}{c}\text { Row } \\
\text { Totals } \\
58,600 \\
6.90\end{array}$} \\
\hline & & \multirow{2}{*}{$\begin{array}{c}65-69 \\
\text { years } \\
8,866 \\
5.04\end{array}$} & \multirow{2}{*}{$\begin{array}{c}70-74 \\
\text { years } \\
10,804 \\
5.02 \\
\end{array}$} & \multirow{2}{*}{$\begin{array}{c}75-79 \\
\text { years } \\
12,030 \\
5.82 \\
\end{array}$} & \multirow{2}{*}{$\begin{array}{c}\begin{array}{c}80-84 \\
\text { years }\end{array} \\
11,601 \\
6.93 \\
\end{array}$} & \multirow{2}{*}{$\begin{array}{c}\text { Over } 84 \\
\text { years } \\
15,299 \\
10.12\end{array}$} & \\
\hline \multirow{5}{*}{ 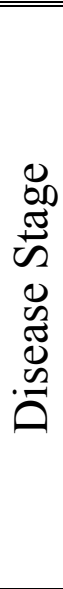 } & $\begin{array}{c}\text { Disease } \\
\text { Stage } 1.1\end{array}$ & & & & & & \\
\hline & $\begin{array}{c}\text { Disease } \\
\text { Stage 1.3- } \\
2.3 \\
\end{array}$ & $\begin{array}{l}906 \\
5.74\end{array}$ & $\begin{array}{l}1061 \\
5.94\end{array}$ & $\begin{array}{c}1,071 \\
6.63\end{array}$ & $\begin{array}{c}944 \\
10.17\end{array}$ & $\begin{array}{l}1,099 \\
10.56\end{array}$ & $\begin{array}{c}5,081 \\
7.83\end{array}$ \\
\hline & $\begin{array}{c}\text { Disease } \\
\text { Stage } 3.1- \\
3.6 \\
\end{array}$ & $\begin{array}{c}696 \\
12.78\end{array}$ & $\begin{array}{c}804 \\
12.81\end{array}$ & $\begin{array}{l}1,068 \\
15.07\end{array}$ & $\begin{array}{l}1,005 \\
16.42\end{array}$ & $\begin{array}{l}1,519 \\
21.92\end{array}$ & $\begin{array}{l}5,092 \\
16.71\end{array}$ \\
\hline & $\begin{array}{c}\text { Disease } \\
\text { Stage } 3.7\end{array}$ & $\begin{array}{l}1,411 \\
15.31\end{array}$ & $\begin{array}{l}1,666 \\
14.89\end{array}$ & $\begin{array}{l}1,771 \\
17.00\end{array}$ & $\begin{array}{l}1,413 \\
22.29\end{array}$ & $\begin{array}{l}1,710 \\
28.12\end{array}$ & $\begin{array}{l}7,971 \\
19.58\end{array}$ \\
\hline & $\begin{array}{c}\text { Disease } \\
\text { Stage } 3.8\end{array}$ & $\begin{array}{c}163 \\
44.79\end{array}$ & $\begin{array}{c}235 \\
42.56\end{array}$ & $\begin{array}{c}226 \\
44.70\end{array}$ & $\begin{array}{c}246 \\
56.10\end{array}$ & $\begin{array}{c}323 \\
53.93\end{array}$ & $\begin{array}{l}1,193 \\
49.12\end{array}$ \\
\hline \multirow{5}{*}{ 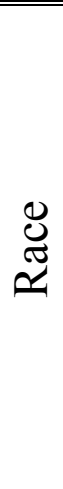 } & White & $\begin{array}{c}7,606 \\
7.19\end{array}$ & $\begin{array}{c}9,900 \\
7.63\end{array}$ & $\begin{array}{c}11,352 \\
8.76\end{array}$ & $\begin{array}{c}11,012 \\
10.43\end{array}$ & $\begin{array}{c}14,734 \\
13.84\end{array}$ & $\begin{array}{c}54,604 \\
10.04\end{array}$ \\
\hline & Black & $\begin{array}{c}1,517 \\
9.76\end{array}$ & $\begin{array}{c}1,413 \\
8.56\end{array}$ & $\begin{array}{c}1,386 \\
7.86\end{array}$ & $\begin{array}{l}1,208 \\
10.59\end{array}$ & $\begin{array}{l}1,446 \\
13.21\end{array}$ & $\begin{array}{l}6,970 \\
10.00\end{array}$ \\
\hline & Hispanic & $\begin{array}{c}2,070 \\
6.33\end{array}$ & $\begin{array}{c}2,079 \\
5.34\end{array}$ & $\begin{array}{c}2,163 \\
6.98\end{array}$ & $\begin{array}{c}2,007 \\
7.82\end{array}$ & $\begin{array}{l}2,752 \\
11.08\end{array}$ & $\begin{array}{c}11,071 \\
7.72\end{array}$ \\
\hline & Asian & $\begin{array}{l}825 \\
6.06\end{array}$ & $\begin{array}{c}1,150 \\
5.91\end{array}$ & $\begin{array}{c}1,240 \\
6.29\end{array}$ & $\begin{array}{l}965 \\
8.08\end{array}$ & $\begin{array}{c}995 \\
11.16\end{array}$ & $\begin{array}{c}5,175 \\
7.44\end{array}$ \\
\hline & $\begin{array}{c}\text { Native } \\
\text { American }\end{array}$ & $\begin{array}{c}24 \\
4.17 \\
\end{array}$ & $\begin{array}{c}28 \\
7.14 \\
\end{array}$ & $\begin{array}{c}25 \\
8.00\end{array}$ & $\begin{array}{c}17 \\
35.29 \\
\end{array}$ & $\begin{array}{c}23 \\
26.09 \\
\end{array}$ & $\begin{array}{c}117 \\
14.53 \\
\end{array}$ \\
\hline \multirow{2}{*}{$\underset{0}{凶}$} & Female & $\begin{array}{c}6,024 \\
6.57 \\
\end{array}$ & $\begin{array}{c}7,338 \\
6.20 \\
\end{array}$ & $\begin{array}{c}8,424 \\
7.28 \\
\end{array}$ & $\begin{array}{c}8,244 \\
9.27 \\
\end{array}$ & $\begin{array}{c}12,422 \\
13.23 \\
\end{array}$ & $\begin{array}{c}42,452 \\
9.12 \\
\end{array}$ \\
\hline & Male & $\begin{array}{c}6,018 \\
7.99\end{array}$ & $\begin{array}{c}7,232 \\
8.32\end{array}$ & $\begin{array}{c}7,742 \\
9.31\end{array}$ & $\begin{array}{l}6,965 \\
10.83\end{array}$ & $\begin{array}{l}7,528 \\
13.39\end{array}$ & $\begin{array}{c}35,485 \\
10.05\end{array}$ \\
\hline \multicolumn{2}{|c|}{ Column Totals } & $\begin{array}{c}12,042 \\
7.28\end{array}$ & $\begin{array}{c}14,570 \\
7.25\end{array}$ & $\begin{array}{c}16,166 \\
8.25\end{array}$ & $\begin{array}{c}15,209 \\
9.98\end{array}$ & $\begin{array}{c}19,950 \\
13.29\end{array}$ & $\begin{array}{c}77,937 \\
9.54\end{array}$ \\
\hline
\end{tabular}

Note: the cell frequency is the top number and the mortality rate (in percentage terms) is the second number in each cell. 
Table 2

Hospital Frequency, Patients Treated, and Mortality

By Hospital Classification

\begin{tabular}{|c||c|c|c|c||c|}
\hline & $\begin{array}{c}\text { Less than } \\
150 \text { Beds }\end{array}$ & $\begin{array}{c}150-200 \\
\text { Beds }\end{array}$ & $\begin{array}{c}200-299 \\
\text { Beds }\end{array}$ & $\begin{array}{c}\text { Over 299 } \\
\text { Beds }\end{array}$ & Row Totals \\
\hline \hline \multirow{2}{*}{$\begin{array}{c}\text { Private, Not- } \\
\text { for-Profit }\end{array}$} & 9 & 4 & 19 & 19 & 51 \\
\hline \multirow{2}{*}{$\begin{array}{c}\text { Private, For- } \\
\text { profit }\end{array}$} & 9,891 & 2,411 & 16,241 & 22,337 & 45,834 \\
& 9.10 & 11.03 & 9.48 & 9.71 & 9.64 \\
\hline & 9.03 & 15 & 7 & 1 & 57 \\
Teaching & & 6,826 & 4,482 & 976 & 22,977 \\
& 0 & 9.52 & 10.46 & 10.55 & 9.52 \\
\hline & & 0 & & 5 & 5 \\
Public & 0 & & 0 & 7,046 & 7,046 \\
& & & & 9.08 & 9.08 \\
\hline \hline \multirow{2}{*}{ Column } & 43 & 19 & 235 & 1,845 & 4 \\
Totals & 15,538 & 9,237 & 20,958 & 32,204 & 77,937 \\
\hline
\end{tabular}

Note: The first number in each cell is the number of hospitals in that category, the second number is the total number of pneumonia patients discharged from hospitals in that cell, and the third number is the morality rate (patient weighted) for patients where were discharged from hospitals in that cell.

${ }^{24}$ Includes all private, not-for-profit hospitals not listed under 'Teaching'.

${ }^{25}$ Includes all private Council Of Teaching Hospitals (COTH) members. 


\section{Table 3}

\section{Cross-tabulation of Disease Stage By Hospital Classification}

\begin{tabular}{|c|c|c|c|c|c|c|c|c|c|}
\hline \multirow[b]{2}{*}{$\begin{array}{c}\text { Severity } \\
\text { Classification }\end{array}$} & \multicolumn{8}{|c|}{ Hospital Categories } & \multirow[b]{2}{*}{$\begin{array}{l}\text { Row } \\
\text { Means }\end{array}$} \\
\hline & $\begin{array}{c}\text { Less } \\
\text { than } \\
150 \\
\text { Beds }\end{array}$ & $\begin{array}{c}150- \\
200 \\
\text { Beds }\end{array}$ & $\begin{array}{c}200- \\
299 \\
\text { Beds }\end{array}$ & $\begin{array}{c}\text { Over } \\
299 \\
\text { Beds }\end{array}$ & $\begin{array}{l}\text { Priv., } \\
\text { Not- } \\
\text { for- } \\
\text { Profit }\end{array}$ & $\begin{array}{l}\text { Priv., } \\
\text { For- } \\
\text { profit }\end{array}$ & $\begin{array}{l}\text { Teach } \\
\text {-ing }\end{array}$ & Public & \\
\hline $\begin{array}{c}\text { Disease } \\
\text { Stage } 1.1\end{array}$ & $0.759^{*}$ & 0.752 & 0.753 & $0.747^{*}$ & 0.751 & $0.741^{*}$ & $0.788^{*}$ & 0.755 & 0.752 \\
\hline $\begin{array}{c}\text { Disease } \\
\text { Stage 1.3-2.3 }\end{array}$ & 0.064 & 0.070 & 0.063 & 0.066 & 0.066 & 0.068 & $0.054^{*}$ & $0.052^{*}$ & 0.065 \\
\hline $\begin{array}{c}\text { Disease } \\
\text { Stage } 3.1-3.6\end{array}$ & $0.059^{*}$ & 0.064 & 0.064 & $0.070^{*}$ & 0.067 & 0.064 & $0.056^{*}$ & 0.072 & 0.065 \\
\hline $\begin{array}{c}\text { Disease } \\
\text { Stage } 3.7 \\
\end{array}$ & 0.103 & 0.101 & 0.104 & 0.101 & 0.100 & $0.111^{*}$ & $0.087^{*}$ & 0.095 & 0.102 \\
\hline $\begin{array}{c}\text { Disease } \\
\text { Stage } 3.8\end{array}$ & 0.014 & 0.014 & 0.015 & 0.016 & 0.015 & 0.015 & 0.014 & $0.026^{*}$ & 0.015 \\
\hline
\end{tabular}

*Significantly different from that category mean at the 5\% level. 


\section{Table $4^{\text {a }}$}

Mortality equation parameter posterior means and standard deviations

$$
\text { Selection model Probit model }
$$

Demographic covariates

$\begin{array}{lrlrr}\text { Age 70-74 } & -0.016 & (0.024) & -0.006 & (0.025) \\ \text { Age 75-79 } & 0.055 & (0.024) & 0.076 & (0.024) \\ \text { Age 80-84 } & 0.171 & (0.024) & 0.199 & (0.024) \\ \text { Age }>84 & 0.351 & (0.022) & 0.382 & (0.023) \\ \text { Female } & -0.083 & (0.013) & -0.084 & (0.013) \\ \text { Black } & -0.024 & (0.027) & -0.034 & (0.027) \\ \text { Hispanic } & -0.124 & (0.022) & -0.115 & (0.023) \\ \text { Native } & 0.161 & (0.132) & 0.210 & (0.153) \\ \text { Asian } & -0.096 & (0.030) & -0.100 & (0.031) \\ \text { Income } & 0.115 & (0.158) & 0.296 & (0.201) \\ \text { Income }^{\wedge 2} & -0.018 & (0.019) & -0.033 & (0.023)\end{array}$

Disease severity covariates

$\begin{array}{lllll}\text { Emergency admit } & 0.019 & (0.019) & 0.176 & (0.016) \\ \text { Disease stages 1.3-2.3 } & 0.094 & (0.028) & 0.096 & (0.028) \\ \text { Disease stages 3.1-3.6 } & 0.491 & (0.022) & 0.507 & (0.023) \\ \text { Disease stage 3.7 } & 0.633 & (0.018) & 0.654 & (0.018) \\ \text { Disease stage 3.8 } & 1.390 & (0.038) & 1.453 & (0.038)\end{array}$

$-q_{j}$ (Negative of hospital group quality probits)

$\begin{array}{lrrrr}\text { Less than } 150 \text { beds } & -0.047 & (0.023) & -0.014 & (0.012) \\ \text { Between 150 and 199 beds } & 0.045 & (0.031) & 0.036 & (0.018) \\ \text { Between 200 and 299 beds } & 0.063 & (0.016) & 0.016 & (0.013) \\ \text { 300 beds or more } & -0.002 & (0.018) & 0.006 & (0.012) \\ \text { Private, not for profit } & 0.029 & (0.012) & 0.012 & (0.010) \\ \text { Private, for profit } & -0.038 & (0.012) & 0.006 & (0.009) \\ \text { Teaching } & 0.041 & (0.035) & -0.011 & (0.023) \\ \text { Public } & 0.087 & (0.079) & 0.014 & (0.042)\end{array}$

${ }^{\mathrm{a}}$ Posterior means are accompanied by posterior standard deviations in parentheses. For the selection model, the function of interest is $\beta_{j} /(\delta \Sigma \delta+1)^{1 / 2}$ and for the probit model it is $\beta_{j} / \sigma$. In the case of the hospital group quality probits, the weights are proportional to the number of patients in the sample for each hospital. 


\section{Table 5}

Posterior Probability Comparisons of Group Hospital Quality Probits, Selection Model ${ }^{\mathrm{a}}$

$$
\begin{aligned}
& \text { A. Hospitals grouped by size } \\
& <150 \text { beds } \quad 150-199 \text { beds } 200-299 \text { beds }
\end{aligned}
$$

150-199 beds

0.993

200-299 beds

1.00

0.678

$\geq 300$ beds

0.889

0.044

0.009

B. Hospitals grouped by ownership classification Private not for profit Private for profit Public

Private for profit $\quad 0.001$

$\begin{array}{lll}\text { Public } & 0.756 & 0.937\end{array}$

$\begin{array}{llll}\text { Teaching } & 0.621 & 0.957 & 0.315\end{array}$

${ }^{a}$ Entries indicate the posterior probability that the group quality probit $q_{G}$ in the column classification exceeds the group quality probit $q_{G}$ in the row classification. 


\section{Table 6}

Expected Mortality Comparisons, from a base of .10, Selection Model ${ }^{\mathrm{a}}$

\begin{tabular}{lllll}
\multicolumn{5}{c}{ A. Hospitals grouped by size } \\
$<150$ beds & 0.100 & $\begin{array}{l}0.118 \\
(0.010)\end{array}$ & $\begin{array}{l}0.121 \\
(0.006)\end{array}$ & $\begin{array}{l}0.108 \\
(0.007)\end{array}$ \\
& & & & \\
$150-159$ beds & 0.086 & 0.100 & 0.104 & 0.092 \\
& $(0.007)$ & & $(0.007)$ & $(0.05)$ \\
& & & & \\
$200-299$ beds & 0.082 & 0.097 & 0.100 & 0.089 \\
& $(0.004)$ & $(0.007)$ & & $(0.004)$ \\
$>=300$ beds & 0.093 & 0.109 & 0.112 & 0.100 \\
& $(0.006)$ & $(0.005)$ & $(0.005)$ &
\end{tabular}

B. Hospitals grouped by ownership classification

$\begin{array}{lllll}\text { Private not for profit } & 0.100 & \begin{array}{l}0.089 \\ (0.004)\end{array} & \begin{array}{l}0.112 \\ (0.015)\end{array} & \begin{array}{l}0.102 \\ (0.006)\end{array} \\ \text { Private for profit } & \begin{array}{l}0.113 \\ (0.004)\end{array} & 0.100 & 0.125 & 0.115 \\ & & (0.016) & (0.007) \\ \text { Public } & 0.091 & 0.089 & 0.100 & 0.093 \\ & (0.013) & (0.0040) & & (0.015) \\ \text { Teaching } & 0.098 & 0.087 & 0.109 & 0.100 \\ & (0.006) & (0.006) & (0.017) & \end{array}$

${ }^{a}$ Cells contain expected mortality at hospitals in the column classification, given a patient with an expected mortality of 0.100 at hospitals in the row classification. 


\section{Table $7^{\mathrm{a}}$}

Choice equation parameter posterior means and standard deviations
Covariate
Distance
$-7.170(0.13 x)$
Distance $^{2}$
$0.732 \quad(0.009)$
Distance $\times$ Age
$-0.815 \quad(0.024)$
Distance $\times$ Severity
$-0.605 \quad(0.035)$
$10^{-5} \times$ Distance $\times$ Income
$-0.848 \quad(0.252)$

${ }^{a}$ Distance is measured in hundreds of kilometers. The age variable takes on the value 1 for ages 65-69, 2 for 70-74, 3 for 75-79, 4 for 80-84, and 5 for 85 and above. The severity variable is disease stage $(1.1 ., 1.2, \ldots 3.8)$. Income is in dollars per year. 


\section{Table 8}

Relations between hospital quality probits and severity correlations in the sample

\begin{tabular}{lcc}
\multicolumn{4}{c}{ A. Moments of $q_{j}, q_{j}^{*}, \rho_{j}$} \\
& Posterior mean & Posterior standard deviation \\
$q_{j}$ & $?$ & .105 \\
$q_{j}^{*}$ & $?$ & .178 \\
$\rho_{j}$ & $?$ & .055
\end{tabular}

Posterior variances and correlations

$\begin{array}{llll}q_{j} & .0109 & .343 & .543 \\ q_{j}^{*} & .0020 & .0031 & -.537 \\ \rho_{j} & .0101 & -.0053 & .0318\end{array}$

(Correlations shown above the main diagonal)

B. OLS regression of $\rho_{j}$ (posterior means) on $q_{j}$ (posterior means)

$$
\begin{aligned}
\rho_{j}= & -.004+.181 q_{j} ; R^{2}=.117, s=.052 \\
& (.005)(.046)
\end{aligned}
$$

C. OLS regression of $q_{j}^{*}$ (posterior means) on $q_{j}$ and $\rho_{j}$ (posterior means)

$$
\begin{aligned}
q_{j}^{*}=-.011+ & 1.404 q_{j}-2.639 \rho_{j} ; \quad R^{2}=.886, s=.061 \\
(.057) \quad(.108) &
\end{aligned}
$$

D. OLS regression of $\rho_{j}$ (posterior means) on $q_{j}$ (posterior means)

$$
q_{j}^{*}=.000+.926 q_{j} ; \quad R^{2}=.295, s=.151
$$




\section{Table 9}

Hospital quality probits and severity correlations for seven hospitals

\section{Hospital}

Community Hospital of Gardena

Pioneer Hospital

Beverly Hospital

San Pedro Peninsula Hospital

Westlake Medical Center

City of Hope National Medical

Linda Vista Community Hospital
Posterior means (standard deviations)

$$
q_{j} \quad \rho_{j} \quad q_{j}^{*}
$$

$\begin{array}{lll}.124(.094) & .124(.052) & -.126(.148) \\ .113(.075) & .137(.040) & -.221(.077) \\ -.014(.065) & -.100(.040) & .012(.055) \\ -.033(.065) & -.117(.035) & .140(.067) \\ .123(.094) & -.132(.051) & .319(.151) \\ -.172(.115) & .122(.050) & -.777(.142) \\ .127(.110) & -.102(.063) & .528(.215)\end{array}$


Table 10

Posterior Distribution of Hospital Quality Probits, Selection model

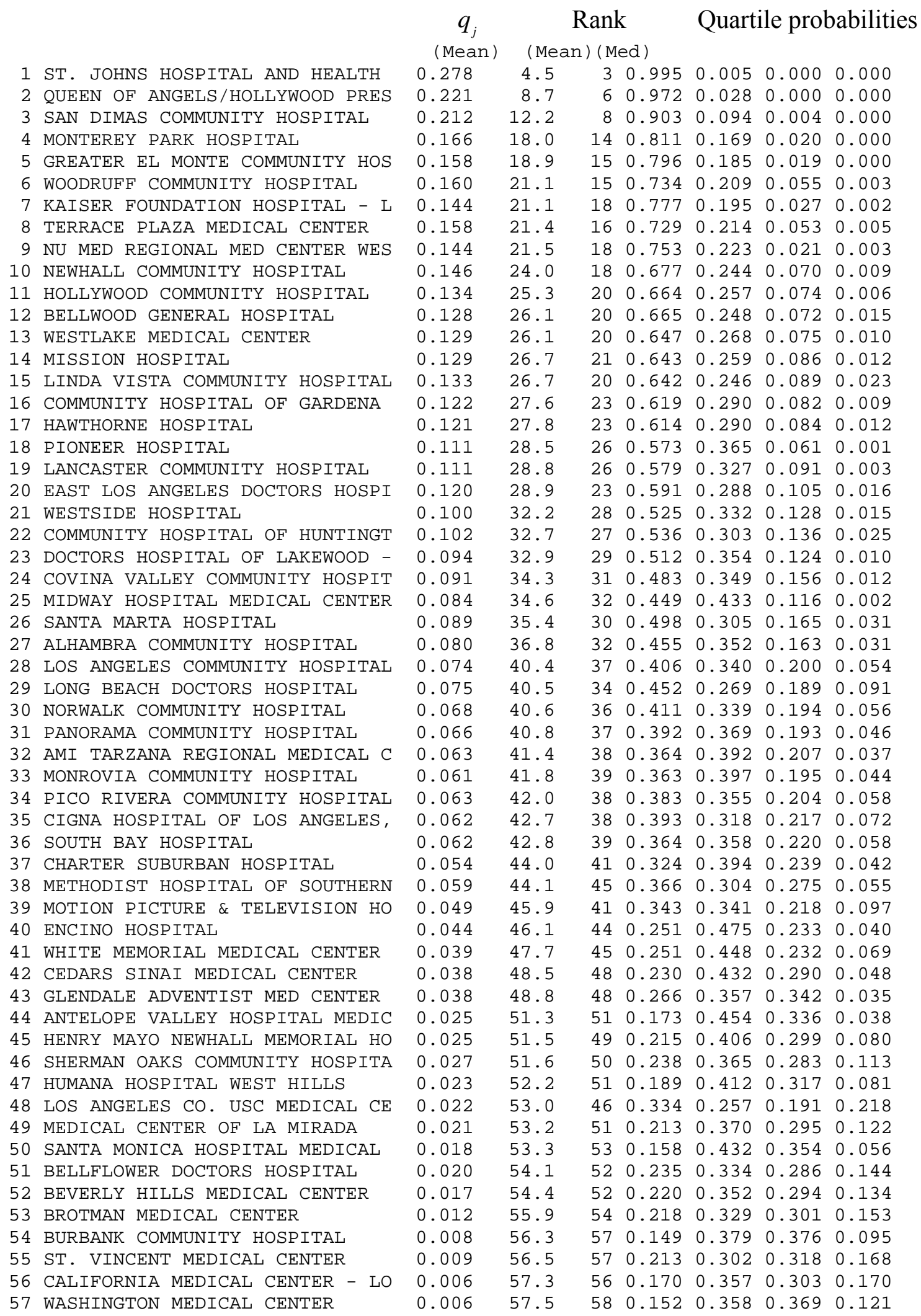




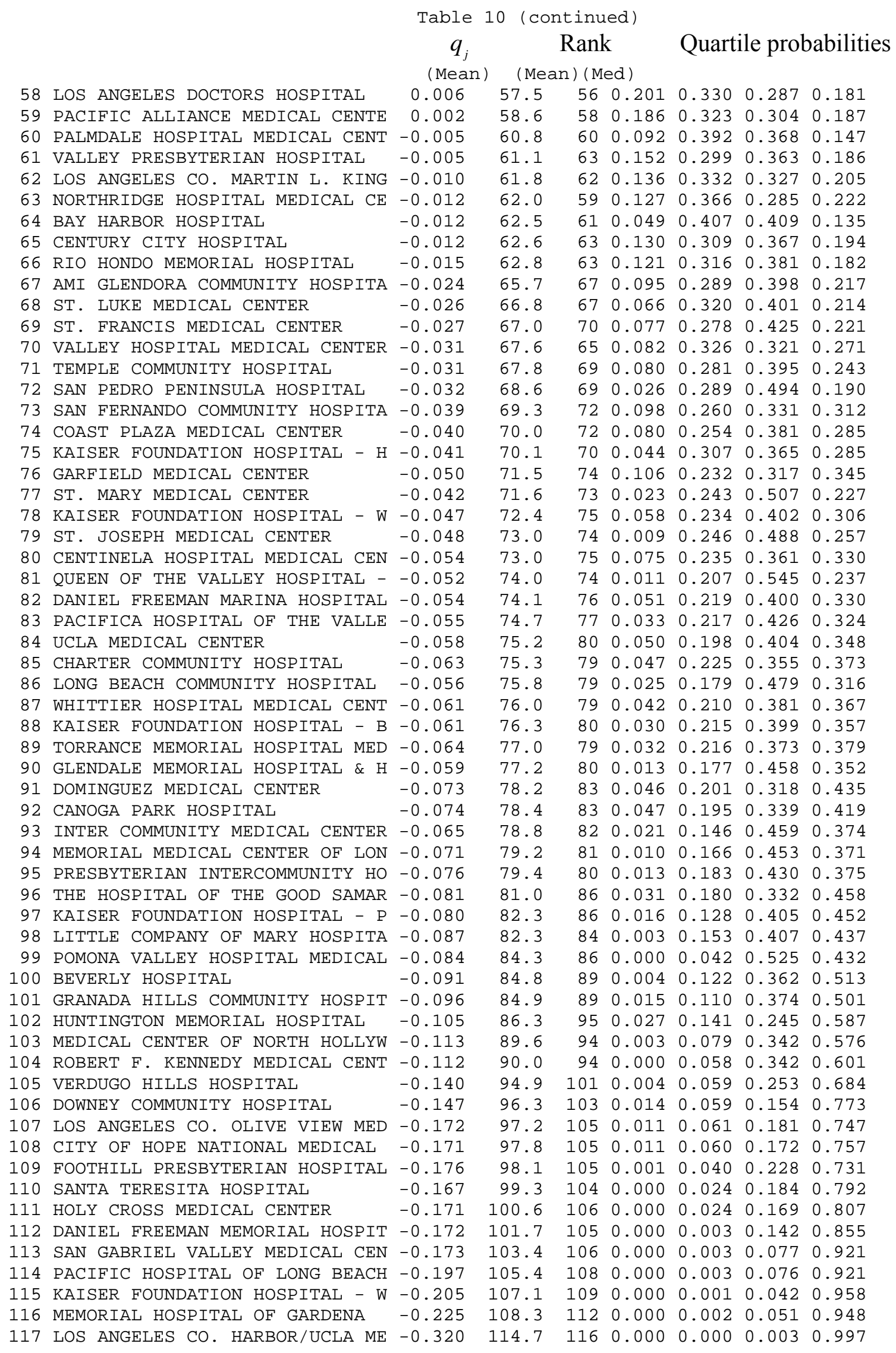


Table 11

\section{Comparison of Selected Hospital Quality Probits, Selection Model}

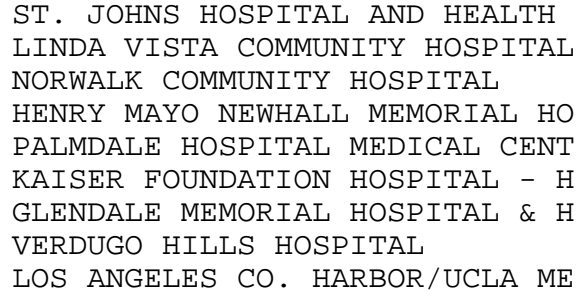

$\begin{array}{rcccccccc} & 1 & 15 & 30 & 45 & 60 & 75 & 90 & 105 \\ 15 & 0.885 & & & & & & \\ 30 & 0.978 & 0.675 & & & & & \\ 45 & 1.000 & 0.801 & 0.623 & & & & \\ 60 & 0.993 & 0.822 & 0.695 & 0.557 & & & \\ 75 & 0.999 & 0.903 & 0.791 & 0.714 & 0.623 & & \\ 90 & 0.997 & 0.894 & 0.807 & 0.696 & 0.722 & 0.566 & & \\ 105 & 1.000 & 0.950 & 0.896 & 0.862 & 0.842 & 0.799 & 0.814 & \\ 117 & 1.000 & 0.999 & 1.000 & 1.000 & 0.997 & 0.986 & 0.990 & 0.881\end{array}$


Table 12

Posterior Distribution of Hospital Quality Probits, Probit model

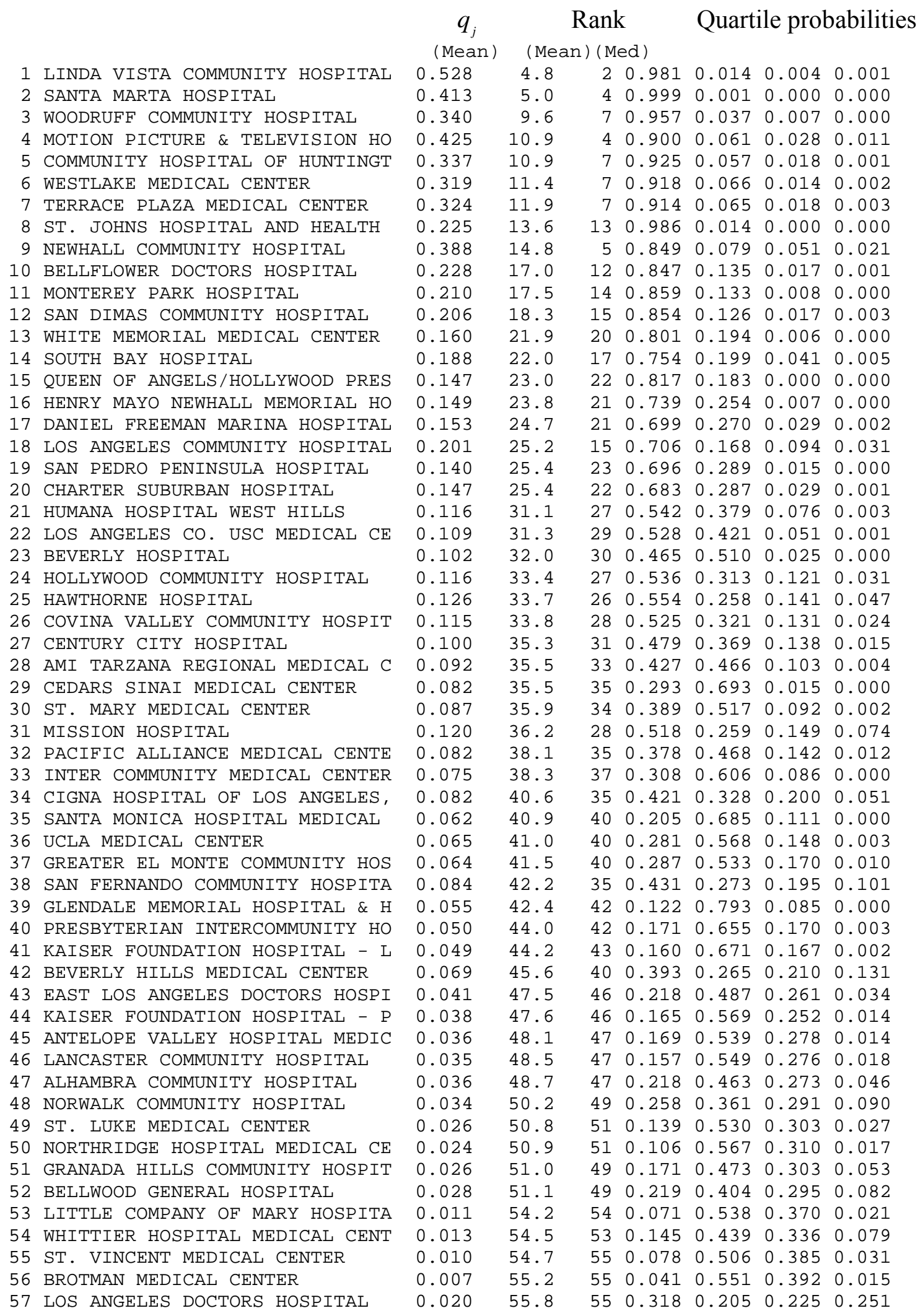


Table 12 (continued)

\begin{tabular}{|c|c|c|c|c|c|c|c|c|}
\hline \multirow[b]{2}{*}{58} & \multirow[b]{2}{*}{ KAISER FOUNDATION HOSPITAL - B } & $\begin{array}{c}q_{j} \\
\text { (Mean) }\end{array}$ & \multicolumn{3}{|c|}{$\begin{array}{c}\text { Rank } \\
(\text { Mean) (Med) }\end{array}$} & \multicolumn{3}{|c|}{ Quartile probabilities } \\
\hline & & 0.002 & 56.8 & 56 & 0.058 & 0.489 & 0.411 & 0.042 \\
\hline 59 & KAISER FOUNDATION HOSPITAL - H & 0.001 & 57.3 & 57 & 0.078 & 0.460 & 0.411 & 0.051 \\
\hline 60 & ST. JOSEPH MEDICAL CENTER & -0.001 & 57.6 & 58 & 0.008 & 0.521 & 0.467 & 0.004 \\
\hline 61 & THE HOSPITAL OF THE GOOD SAMAR & -0.004 & 58.2 & 58 & 0.023 & 0.480 & 0.474 & 0.022 \\
\hline 62 & LOS ANGELES CO. MARTIN L. KING & -0.006 & 59.2 & 59 & 0.103 & 0.393 & 0.394 & 0.111 \\
\hline 63 & TORRANCE MEMORIAL HOSPITAL MED & -0.008 & 59.2 & 59 & 0.037 & 0.450 & 0.465 & 0.049 \\
\hline 64 & MEMORIAL MEDICAL CENTER OF LON & -0.008 & 59.5 & 60 & 0.024 & 0.449 & 0.497 & 0.030 \\
\hline 65 & ROBERT F. KENNEDY MEDICAL CENT & -0.020 & 62.5 & 63 & 0.039 & 0.365 & 0.521 & 0.075 \\
\hline 66 & NU MED REGIONAL MED CENTER WES & -0.024 & 63.7 & 63 & 0.038 & 0.367 & 0.486 & 0.110 \\
\hline 67 & LOS ANGELES CO. OLIVE VIEW MED & -0.027 & 64.1 & 65 & 0.144 & 0.285 & 0.334 & 0.237 \\
\hline 68 & SAN GABRIEL VALLEY MEDICAL CEN & -0.026 & 64.2 & 65 & 0.006 & 0.319 & 0.644 & 0.030 \\
\hline 69 & DOCTORS HOSPITAL OF LAKEWOOD - & -0.029 & 64.9 & 65 & 0.017 & 0.327 & 0.583 & 0.074 \\
\hline 70 & AMI GLENDORA COMMUNITY HOSPITA & -0.033 & 65.8 & 68 & 0.120 & 0.282 & 0.345 & 0.253 \\
\hline 71 & LONG BEACH DOCTORS HOSPITAL & -0.051 & 69.9 & 73 & 0.093 & 0.247 & 0.361 & 0.298 \\
\hline 72 & SHERMAN OAKS COMMUNITY HOSPITA & -0.055 & 70.9 & 73 & 0.066 & 0.246 & & 0.291 \\
\hline 73 & GLENDALE ADVENTIST MED CENTER & -0.052 & 71.6 & 72 & 0.000 & 0.130 & 0.809 & 0.061 \\
\hline 74 & HOLY CROSS MEDICAL CENTER & -0.055 & 71.9 & 73 & 0.004 & 0.214 & 0.623 & 0.159 \\
\hline 75 & GARFIELD MEDICAL CENTER & -0.054 & 72.0 & 73 & 0.002 & 0.167 & 0.718 & 0.114 \\
\hline 76 & ENCINO HOSPITAL & -0.062 & 72.9 & 75 & 0.037 & 0.222 & 0.478 & 0.263 \\
\hline 77 & METHODIST HOSPITAL OF SOUTHERN & -0.063 & 74.3 & 75 & 0.000 & 0.105 & 0.760 & 0.135 \\
\hline 78 & BURBANK COMMUNITY HOSPITAL & -0.073 & 75.4 & 78 & 0.029 & 0.206 & 0.436 & 0.329 \\
\hline 79 & KAISER FOUNDATION HOSPITAL - W & -0.074 & 76.3 & 78 & 0.013 & 0.175 & & 0.298 \\
\hline 80 & CENTINELA HOSPITAL MEDICAL CEN & -0.075 & 76.6 & 78 & 0.007 & 0.160 & 0.551 & 0.281 \\
\hline 81 & WESTSIDE HOSPITAL & -0.079 & 77.3 & 80 & 0.014 & 0.173 & 0.484 & 0.329 \\
\hline 82 & PALMDALE HOSPITAL MEDICAL CENT & -0.086 & 78.1 & 82 & 0.022 & 0.181 & 0.417 & 380 \\
\hline 83 & VALLEY PRESBYTERIAN HOSPITAL & -0.079 & 78.4 & 79 & 0.000 & 0.093 & 0.653 & 0.255 \\
\hline 84 & MONROVIA COMMUNITY HOSPITAL & -0.088 & 78.4 & 83 & 0.034 & 0.179 & 0.389 & 0.399 \\
\hline 85 & MEDICAL CENTER OF LA MIRADA & -0.109 & 82.0 & 88 & 0.042 & 0.150 & 0.317 & \\
\hline 86 & PACIFIC HOSPITAL OF LONG BEACH & -0.098 & 82.3 & 84 & 03 & 0.100 & 492 & 05 \\
\hline 87 & PICO RIVERA COMMUNITY HOSPITAL & -0.129 & 82.8 & 92 & 0.073 & 0.150 & 0.232 & 545 \\
\hline 88 & WASHINGTON MEDICAL CENTER & -0.105 & 83.2 & 86 & 0.011 & 0.095 & 0.440 & 0.453 \\
\hline 89 & COMMUNITY HOSPITAL OF GARDENA & -0.126 & 83.4 & 91 & 064 & 0.139 & 0.270 & 0.527 \\
\hline 90 & HUNTINGTON MEMORIAL HOSPITAL & -0.099 & 83.8 & 85 & 0.000 & 0.018 & 0.626 & 0.356 \\
\hline 91 & FOOTHILL PRESBYTERIAN HOSPITAL & -0.107 & 84 . & 86 & 0.000 & 0.071 & 0.474 & 0.455 \\
\hline 92 & MEDICAL CENTER OF NORTH HOLLYW & -0.109 & 85.5 & 87 & 0.001 & 0.034 & 18 & 47 \\
\hline 93 & SANTA TERESITA HOSPITAL & -0.113 & 86.1 & 88 & 0.001 & 0.045 & 0.470 & 0.485 \\
\hline 94 & DOWNEY COMMUNITY HOSPITAL & -0.112 & 86.6 & 88 & 0.000 & 0.022 & 0.486 & 0.491 \\
\hline 95 & TEMPLE COMMUNITY HOSPITAL & -0.131 & 87.3 & 92 & 0.012 & 0.101 & 0.330 & 0.558 \\
\hline 96 & VALLEY HOSPITAL MEDICAL CENTER & -0.124 & 88.3 & 90 & 0.000 & 0.037 & 0.415 & 0.548 \\
\hline 97 & BAY HARBOR HOSPITAL & -0.126 & 89.7 & 91 & 0.000 & 0.010 & 0.405 & 0.585 \\
\hline 98 & MIDWAY HOSPITAL MEDICAL CENTER & -0.125 & 89.8 & 91 & 0.000 & 0.003 & 0.410 & \\
\hline 99 & QUEEN OF THE VALLEY HOSPITAL - & -0.128 & 89.9 & 92 & 0.000 & 0.014 & 395 & \\
\hline 100 & CHARTER COMMUNITY HOSPITAL & -0.135 & 91.5 & 93 & 0.000 & 0.010 & 0.339 & 650 \\
\hline 101 & PACIFICA HOSPITAL OF THE VALLE & -0.147 & 91.6 & 96 & 002 & 0.059 & 0.303 & 0.637 \\
\hline 102 & LONG BEACH COMMUNITY HOSPITAL & -0.142 & 92.8 & 95 & 0.000 & 0.009 & 0.309 & 0.683 \\
\hline 103 & VERDUGO HILLS HOSPITAL & -0.149 & 93.9 & 97 & 0.000 & 0.012 & 0.275 & 0.713 \\
\hline 104 & DANIEL FREEMAN MEMORIAL HOSPIT & -0.147 & 93.9 & 96 & 0.000 & 0.006 & 0.272 & 0.722 \\
\hline 105 & POMONA VALLEY HOSPITAL MEDICAL & -0.144 & 94.2 & 95 & 000 & 0.001 & 37 & 0.762 \\
\hline 106 & PANORAMA COMMUNITY HOSPITAL & -0.173 & 95.5 & 101 & 0.003 & 0.039 & 0.229 & 0.729 \\
\hline 107 & RIO HONDO MEMORIAL HOSPITAL & -0.209 & 102.0 & 105 & 0.000 & 0.006 & 0.113 & 0.881 \\
\hline 108 & KAISER FOUNDATION HOSPITAL - W & -0.213 & 103.9 & 106 & 0.000 & 0.001 & 0.054 & 0.945 \\
\hline 109 & PIONEER HOSPITAL & -0.221 & 103.9 & 107 & 0.000 & 0.004 & 0.074 & 0.922 \\
\hline 110 & COAST PLAZA MEDICAL CENTER & -0.256 & 105.4 & 110 & 0.001 & 0.010 & 0.076 & 0.914 \\
\hline 111 & ST. FRANCIS MEDICAL CENTER & -0.221 & 105.7 & 107 & 0.000 & 0.000 & 0.015 & 0.985 \\
\hline 112 & LOS ANGELES CO. HARBOR/UCLA ME & -0.243 & 106.3 & 109 & 0.000 & 0.003 & 0.038 & 0.959 \\
\hline 113 & MEMORIAL HOSPITAL OF GARDENA & -0.250 & 106.6 & 109 & 0.000 & 0.001 & 0.043 & 0.956 \\
\hline 114 & DOMINGUEZ MEDICAL CENTER & -0.348 & 110.1 & 114 & 0.000 & 0.006 & 0.038 & 0.957 \\
\hline 115 & CALIFORNIA MEDICAL CENTER - LO & -0.366 & 113.8 & 114 & 0.000 & 0.000 & 0.000 & 1.000 \\
\hline 116 & CANOGA PARK HOSPITAL & -0.504 & 114.6 & 116 & 0.000 & 0.001 & 0.006 & 0.993 \\
\hline 117 & CITY OF HOPE NATIONAL MEDICAL & -0.777 & 116.9 & 117 & 0.000 & 0.000 & 0.000 & 1.000 \\
\hline
\end{tabular}


Table 13

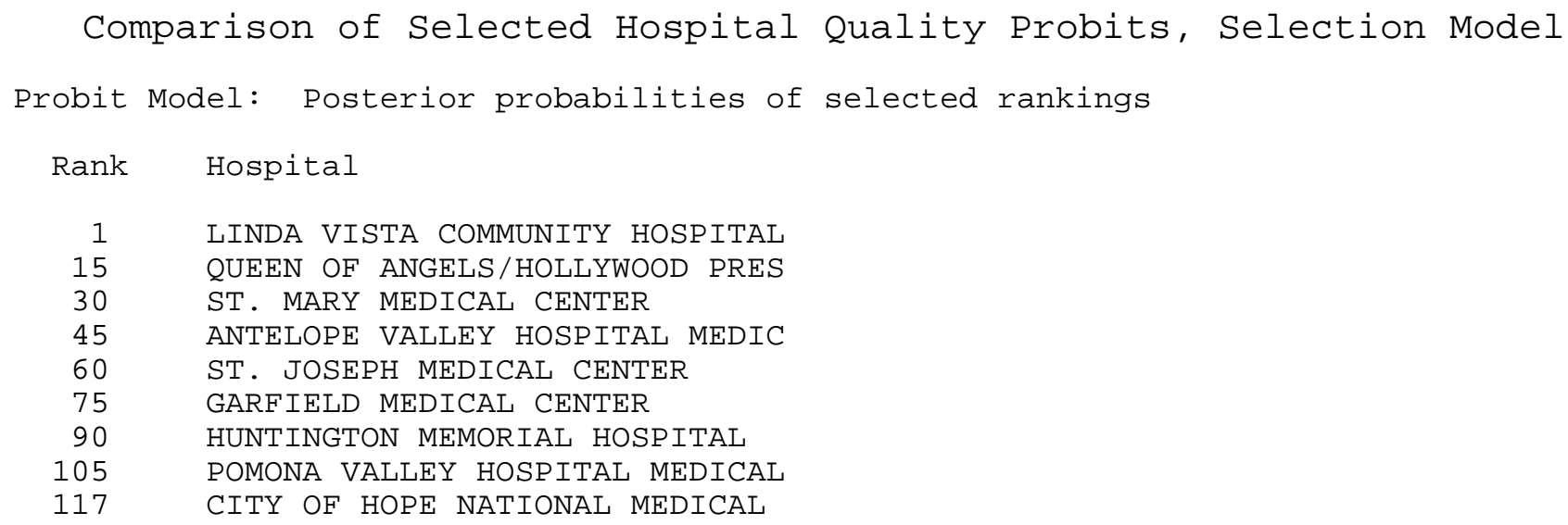

Posterior probability that hospital with rank in row ranks below hospital with rank in column

$\begin{array}{rcccccccc} & 1 & 15 & 30 & 45 & 60 & 75 & 90 & 105 \\ 15 & 0.963 & & & & & & & \\ 30 & 0.979 & 0.768 & & & & & & \\ 45 & 0.990 & 0.914 & 0.703 & & & & \\ 60 & 0.995 & 0.990 & 0.860 & 0.665 & & & \\ 75 & 0.996 & 0.998 & 0.954 & 0.856 & 0.786 & & & \\ 90 & 0.998 & 1.000 & 0.985 & 0.946 & 0.945 & 0.755 & & \\ 105 & 0.999 & 1.000 & 0.997 & 0.987 & 0.995 & 0.920 & 0.775 & \\ 117 & 1.000 & 1.000 & 1.000 & 1.000 & 1.000 & 1.000 & 1.000 & 1.000\end{array}$


Figure 1

Scatterplot of Hospital Quality, $q_{j}$, versus Hospital Severity, $\rho_{j}$

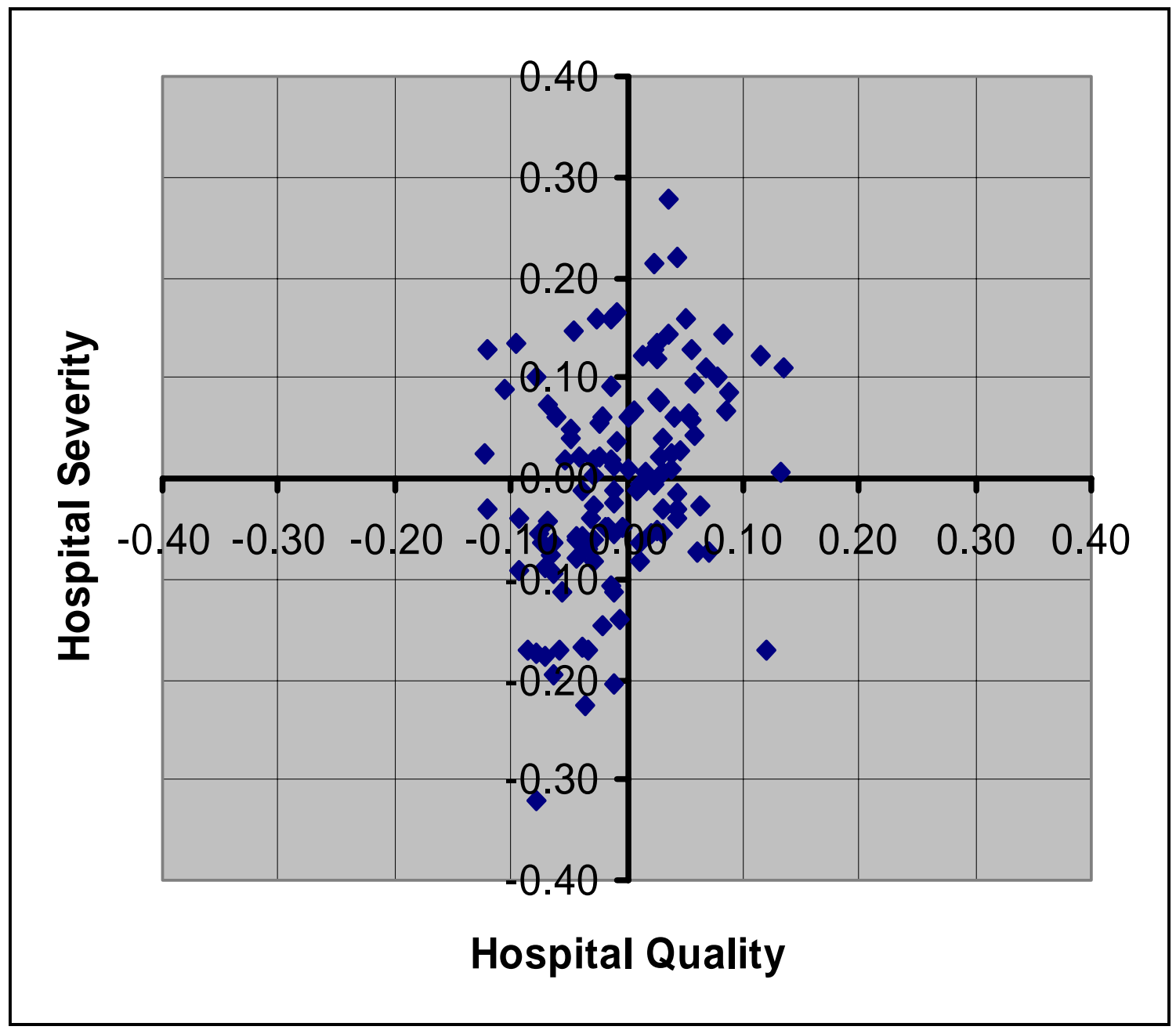




\section{References}

Albert and Chib, 1993, "Bayesian Analysis of Binary and Polychotomous Response Data," Journal of the American Statistical Association, 422, 669-79.

Bernardo and Smith (1996)

Burns L, Wholey D., 1992, "The Impact of Physician Characteristics in Conditional Choice Models for Hospital Care," Journal of Health Economics 11, 43-62.

Chib, S., 1995, "Marginal Likelihood from the Gibbs Output," Journal of the American Statistical Association 90, 1313-1321.

Chib, S., and Greenberg, 1996, "Markov Chain Monte Carlo Simulation Methods in Econometrics," Econometric Theory, 12, 409-31.

Cutler, D. M., 1995, "The Incidence of Adverse Medical Outcomes Under Prospective Payment," Econometrica, 63(1): 29-50.

Fine, J.M., J.D. Scinto, D.H. Galusha, M.K. Petrillo, T.P. Meehan, 1998, "Patient and Hospital Characteristics Associated with Timely Care of Elderly Patients Hospitalized with Pneumonia: Results from the Medicare Quality Indication System Pneumonia Module," Abstract Book, Association of Health Services Research, 15.

Gelfand, A.E., and A.F.M. Smith, 1990, "Sampling Based Approaches to Calculating Marginal Densities," Journal of the American Statistical Association 85: 398-409.

Geweke, J., 1991, "Efficient Simulation from the Multivariate Normal and Student- $t$ Distributions Subject to Linear Constraints," in E. M. Keramidas (ed.), Computing Science and Statistics: Proceedings of the 23rd Symposium on the Interface, 571-578. Fairfax, VA: Interface Foundation of North America.

Geweke, J., 1997, "Posterior Simulators in Econometrics," in D. Kreps and K.F Wallis (eds.), Advances in Economics and Econometrics: Theory and Applications, vol. III. Cambridge: Cambridge University Press, 128-165.

Geweke, J., 1999, "Using Simulation Methods for Bayesian Econometric Models: Inference, Development and Communication," (with discussion and rejoinder) Econometric Reviews 18: $1-126$

Geweke, J., M. Keane and D. Runkle, 1997, "Statistical Inference in the Multinomial, Multiperiod Probit Model," Journal of Econometrics, 80, 125-65.

Gowrisankaran, G., and R.J. Town, 1999, "Estimating the Quality of Care in Hospitals Using Instrumental Variables," Journal of Health Economics 18, 747-67. 
Gray, B., 1991, The Profit Motive and Patient Care, Cambridge, MA: Harvard University Press.

Iezzoni, L.I., 1997, Risk Adjustment for Measuring Health Care Outcomes, Ann Arbor, MI: Health Administration Press, $2^{\text {nd }}$ Edition.

Iezzoni, L.I. et al., 1996, "Severity Measurement Methods and Judging Hospital Death Rates for Pneumonia," Medical Care, 34(1): 11-28.

Keeler, E. B., K.L. Kahn, D. Draper, M.J. Sherwood, L.V. Rubenstein, E.J. Reinisch, J. Kosecoff, and R.H. Brook, 1990, "Changes in Sickness at Admission Following the Introduction of the Prospective Payment System." Journal of the American Medical Association, 264, 1962-68.

Keeler, E. B., et al., 1992, "Hospital Characteristics and Quality of Care." Journal of the American Medical Association, 268 (13): 1709-14.

Kessler, D. and McClellan, 2000, "Is Hospital Competition Socially Wasteful?" Quarterly Journal of Economics, forthcoming.

Lohr, K.N., ed., 1990, Medicare: A Strategy for Quality Assurance. Volume I. Washington DC: National Academy Press.

Luft, H., et al., 1990, “Does Quality Influence Choice of Hospital?” Journal of the American Medical Association 263, 2899-2906.

McClellan M, B. McNeil, and J. Newhouse, 1994, "Does More Intensive Treatment of Acute Myocardial Infarction in the Elderly Reduce Mortality?" Journal of the American Medical Association. 272, 859-866.

McClellan, M. and H. Noguchi, 1998, "Technological Change in Heart-Disease Treatment: Does High Tech Mean Low Value," American Economic Review (Papers and Proceedings), 88 (2): $90-96$.

McClellan M., and D. Staiger, 1999a, "Comparing Hospital Quality at For-Profit and Not-ForProfit Hospitals,” NBER Working Paper 7324.

McClellan M., and D. Staiger, 1999b, “The Quality of Health Care Providers,” NBER Working Paper 7327.

McGarvey, R. and J. Harper, 1993, "Pneumonia Mortality Reduction and Quality Improvement in a Community Hospital," Quality Review Bulletin, 19, 124-30.

Norton, Edward C. and Douglas O. Staiger, 1994 "How Hospital Ownership Affects Access to Care for the Uninsured," RAND Journal of Economics 25, 171-85.

Pennington, J., 1994, Respiratory Infections: Diagnosis and Management, New York: Raven Press, $3^{\text {rd }}$ edition. 
Rello, J. and J. Valles, 1998, "Mortality as an Outcome in Hospital-Acquired Pneumonia," Infection Control and Hospital Epidemiology, 19(10):795-7.

Roberts, G.O., and A.F.M. Smith, 1994, "Simple Conditions for the Convergence of the Gibbs Sampler and Metropolis-Hastings Algorithms," Stochastic Processes and Their Applications 49: 207-216.

Thomas, J.W. and M.L.F. Ashcroft, 1991, "Measuring severity of illness: Six severity systems and their ability to explain cost-variations," Inquiry, 28, 39-55.

United States General Accounting Office, 1994, "Report Cards" Are Useful But Significant Issues Need to be Addressed. (GAO/HEHS-94-219) Washington, DC: United States General Accounting Office.

Wray, N. J. Hollingsworth, N. Petersen, and C. Ashton, 1997, "Case-Mix Adjustment Using Administrative Databases: A Paradigm to Guide Future Research," Medical Care Research and Review, 54, 326-356. 
Appendix: Likelihood function, posterior density, Gibbs sampling algorithm and computation.

Notation. The notation in this appendix is the same as in the paper. We collect all the definitions here for reference, and introduce some additional useful notation.

Indexing:

$$
\begin{array}{ll}
i=1, \ldots, n & \text { Patients in sample } \\
j=1, \ldots, J & \text { Hospitals in California }
\end{array}
$$

Observed variables:

$$
\begin{array}{ll}
m_{i} & \text { Mortality indicator, } 1 \text { if patient dies, else } 0 \\
\mathbf{c}_{i}: J \times 1 & \text { Hospital choice indicator, } c_{i j}=1 \text { if } i \text { chooses } j, \text { else } 0 \\
\mathbf{x}_{i}: k \times 1 & \text { Individual characteristics affecting mortality } \\
\mathbf{Z}_{i}:(J-1) \times q & \text { Individual characteristics affecting hospital choice } \\
\mathbf{W}: J \times r & \text { Matrix of hospital characteristics }(r \geq J) \\
\mathbf{u}_{i}^{\prime} \equiv\left(\mathbf{c}_{i}^{\prime} \mathbf{W}, \mathbf{x}_{i}^{\prime}\right) &
\end{array}
$$

Latent variables

$$
\begin{array}{ll}
m_{i}^{*} & \text { Mortality probit } \\
\mathbf{c}_{i}^{*}:(J-1) \times 1 & \text { Hospital choice probit }
\end{array}
$$

Miscellaneous:

$$
\begin{array}{ll}
\chi_{S}(z) & \text { Indicator function, } \chi_{S}(z)=1 \text { if } z \in S, \text { else } 0 \\
\mathbf{e}_{n}: n \times 1 & \mathbf{e}_{n}^{\prime}=(1, \ldots, 1)
\end{array}
$$

Model. The model for the latent variables and observables is

$$
\begin{aligned}
& \mathbf{c}_{i}^{*}=\mathbf{Z}_{i} \alpha+\eta_{i}, \quad c_{i j}^{*} \equiv 0 \\
& c_{i j}=\prod_{\ell=1}^{J} \chi_{[0, \infty)}\left(c_{i j}^{*}-c_{i \ell}^{*}\right) \\
& m_{i}^{*}=\mathbf{c}_{i}^{\prime} \mathbf{W} \beta+\mathbf{x}_{i}^{\prime} \gamma+\eta_{i}^{\prime} \boldsymbol{\delta}+\zeta_{i} \\
& m_{i}=\chi_{[0, \infty)}\left(m_{i}^{*}\right) \\
& \left(\begin{array}{c}
\eta_{i} \\
\zeta_{i}
\end{array}\right) \stackrel{I I D}{\sim}\left(\mathbf{0},\left[\begin{array}{ll}
\Sigma & \mathbf{0} \\
\mathbf{0}^{\prime} & 1
\end{array}\right]\right) ; \Sigma=\mathbf{I}_{J-1}+\mathbf{e}_{(J-1)} \mathbf{e}_{(J-1)}^{\prime} \\
& \lambda^{\prime} \equiv\left(\beta^{\prime}, \gamma^{\prime}\right), v^{\prime} \equiv\left(\lambda^{\prime}, \delta^{\prime}\right)
\end{aligned}
$$

Prior distribution. As motivated in the text the prior distribution consists of three, independent components: $\lambda \sim \mathrm{N}\left(\underline{\lambda}, \underline{\mathbf{H}}_{\lambda}^{-1}\right) ; \delta \sim \mathrm{N}\left(\underline{\delta}, \underline{\mathbf{H}}_{\delta}^{-1}\right) ; \quad \alpha \sim \mathrm{N}\left(\underline{\alpha}, \underline{\mathbf{H}}_{\alpha}^{-1}\right)$. Hence the prior density is 


$$
\begin{aligned}
& \mathrm{p}(\lambda, \delta, \alpha)=(2 \pi)^{-(r+++J+q-1) / 2}\left|\underline{\mathbf{H}}_{\lambda}\right|^{1 / 2}\left|\underline{\mathbf{H}}_{\delta}\right|^{1 / 2}\left|\underline{\mathbf{H}}_{\alpha}\right|^{1 / 2} \\
& \quad \cdot \exp \left\{-.5\left[(\lambda-\underline{\lambda})^{\prime} \underline{\mathbf{H}}_{\lambda}(\lambda-\underline{\lambda})+(\delta-\underline{\delta}) \underline{\mathbf{H}}_{\delta}(\delta-\underline{\delta})+(\alpha-\underline{\alpha}) \underline{\mathbf{H}}_{\alpha}(\alpha-\underline{\alpha})\right]\right\} .
\end{aligned}
$$

Distribution of observables and latent variables. To derive the joint density of the observable data and latent variables for individual $I$, let $\Phi_{i} \equiv\left\{\mathbf{Z}_{i}, \mathbf{W}, \alpha, \lambda, \delta, \Sigma\right\}$. Then

$$
\begin{gathered}
\mathrm{p}\left(\mathbf{c}_{i}^{*}, \mathbf{c}_{i}, \mathbf{m}_{i}^{*}, \mathbf{m}_{i} \mid \Phi_{i}\right)=\mathrm{p}\left(\mathbf{c}_{i}^{*} \mid \Phi_{i}\right) \mathrm{p}\left(\mathbf{c}_{i} \mid \mathbf{c}_{i}^{*}, \Phi_{i}\right) \mathrm{p}\left(m_{i}^{*} \mid \mathbf{c}_{i}, \mathbf{c}_{i}^{*}, \Phi_{i}\right) \mathrm{p}\left(m_{i} \mid m_{i}^{*}, \mathbf{c}_{\mathbf{c}_{i}}^{*}, \Phi_{i}\right) \\
=\mathrm{p}\left(\mathbf{c}_{i}^{*} \mid \mathbf{Z}_{i}, \alpha, \Sigma\right) \mathrm{p}\left(\mathbf{c}_{i} \mid \mathbf{c}_{i}^{*}\right) \mathrm{p}\left(m_{i}^{*} \mid \mathbf{c}_{i}, \mathbf{c}_{i}^{*}, \mathbf{Z}_{i}, \mathbf{W}, \alpha, \lambda, \delta, \Sigma\right) \mathrm{p}\left(m_{i} \mid m_{i}^{*}\right) \\
=(2 \pi)^{-J / 2}|\Sigma|^{-1 / 2} \exp \left[-.5\left(\mathbf{c}_{i}^{*}-\mathbf{Z}_{i} \alpha\right)^{\prime} \Sigma^{-1}\left(\mathbf{c}_{i}^{*}-\mathbf{Z}_{i} \alpha\right)\right] \cdot\left[\sum_{j=1}^{J} c_{i j} \prod_{\ell=1}^{J} \chi_{[0, \infty)}\left(c_{i j}^{*}-c_{i \ell}^{*}\right)\right] \\
\cdot \exp \left\{-.5\left[m_{i}^{*}-\mathbf{u}_{i}^{\prime} \lambda-\left(\mathbf{c}_{i}^{*}-\mathbf{Z}_{i} \alpha\right)^{\prime} \delta\right]^{2}\right\} \cdot\left[m_{i} \chi_{[0, \infty)}\left(m_{i}^{*}\right)+\left(1-m_{i}\right) \chi_{(-\infty, 0)}\left(m_{i}^{*}\right)\right]
\end{gathered}
$$

Since individuals are independent, the joint distribution of observables and latent variables for all individuals is the product of this expression over $i=1, \ldots, n$.

\section{Gibbs sampling algorithm.}

The posterior density is proportional to the product of the prior density (1) and the distribution of observables and latent variables (2) over $i=1, \ldots, n$, taking the observables as fixed and the unobserved latent variables and parameters as the arguments of the posterior density. In a Gibbs sampling algorithm (Gelfand and Smith, 1990; Geweke, 1996) the unobservables are grouped and successive drawings are made for each group. Given weak regularity conditions, the unique stationary distribution of these repeated drawings is the posterior distribution. In the algorithm described here there are $2 n+2$ groups: $\mathbf{c}_{i}^{*}(i=1, \ldots, n)$, $m_{i}^{*}(i=1, \ldots, n), \alpha$, and $v$. In each case the conditional distribution may be determined by examining the kernel of the posterior density in the vector being drawn.

The latent vectors $\mathbf{c}_{i}^{*}(i=1, \ldots, n)$ are conditionally independent, with $\mathbf{c}_{i}^{*} \sim \mathrm{N}\left(\overline{\mathbf{c}}_{i}, \overline{\mathbf{H}}_{i}^{-1}\right)$ where

$$
\overline{\mathbf{H}}_{i}=\Sigma^{-1}+\delta \delta^{\prime}, \overline{\mathbf{c}}_{i}=\overline{\mathbf{H}}_{i}^{-1}\left[\Sigma^{-1} \mathbf{Z}_{i} \alpha+\delta\left(m_{i}^{*}-\mathbf{u}_{i}^{\prime} \lambda+\delta^{\prime} \mathbf{Z}_{i} \alpha\right)\right],
$$

and subject to $c_{i j}^{*}-c_{i \ell}^{*} \geq 0$ where $j: c_{i j}=1$. While the elements of $\mathbf{c}_{i}^{*}$ can be drawn in succession using the generic algorithm in Geweke (1991), the fixed structure of $\Sigma$ permits a more efficient procedure. Specifically, it can be shown that conditional on all the other parameters and latent variables, the $j^{\prime}$ th element of $\mathbf{c}_{i}^{*}$, denoted $c_{i j}^{*}$, is

$$
c_{i j}^{*} \sim \mathrm{N}\left\{\hat{\boldsymbol{c}}_{i j}+\left(1-J^{-1}+\delta_{j}^{2}\right)^{-1}\left[\sum_{\ell \neq j}\left(J^{-1}-\delta_{j} \delta_{\ell}\right)\left(c_{i \ell}^{*}-\bar{c}_{i \ell}\right)\right]\left(1-J^{-1}+\delta_{j}^{2}\right)^{-1}\right\},
$$


truncated to the interval $(0, \infty) \cap\left(\max _{\ell \neq j} c_{i \ell}^{*}, \infty\right)$ if $j$ is the observed choice; truncated to the interval $\left(-\infty, c_{i k}^{*}\right)$ if $k(\neq j, J)$ is the observed choice; and to $(-\infty, 0)$ if $k=J$ is the observed choice.

The latent vectors $m_{i}^{*}(i=1, \ldots, n)$ are conditionally independent, with $m_{i}^{*} \sim \mathrm{N}\left[\mathbf{u}_{i}^{\prime} \lambda+\delta^{\prime}\left(c_{i}^{*}-\mathbf{Z}_{i} \alpha\right), 1\right]$ subject to $\left(2 m_{i}-1\right) m_{i}^{*} \geq 0$.

The conditional distribution of $\alpha$ is $\alpha \sim \mathrm{N}\left(\bar{\alpha}, \overline{\mathbf{H}}_{\alpha}^{-1}\right)$ where

$$
\begin{aligned}
& \overline{\mathbf{H}}_{\alpha}=\underline{\mathbf{H}}_{\alpha}+\sum_{i=1}^{n} \mathbf{Z}_{i}^{\prime}\left(\Sigma^{-1}+\delta \delta^{\prime}\right) \mathbf{Z}_{i} \\
& \bar{\alpha}=\underline{\mathbf{H}}_{\alpha}^{-1}\left\{\underline{\mathbf{H}}_{\alpha} \underline{\alpha}+\sum_{i=1}^{n} \mathbf{Z}_{i}^{\prime}\left[\Sigma^{-1} \mathbf{c}_{i}^{*}+\delta\left(\delta^{\prime} \mathbf{c}_{i}^{*}-m_{i}^{*}+\mathbf{u}_{i}^{\prime} \lambda\right)\right]\right\} .
\end{aligned}
$$

Let $\underline{v}^{\prime}=\left(\underline{\lambda}^{\prime}, \underline{\delta}\right)$. The conditional distribution of $v$ is $v \sim \mathrm{N}\left(\bar{v}, \overline{\mathbf{H}}_{v}^{-1}\right)$ where

$$
\begin{aligned}
& \overline{\mathbf{H}}_{v}=\left[\begin{array}{cc}
\underline{\mathbf{H}}_{\lambda} & \mathbf{0} \\
\mathbf{0} & \underline{\mathbf{H}}_{\delta}
\end{array}\right]+\sum_{i=1}^{n}\left[\begin{array}{c}
\mathbf{u}_{i} \\
\mathbf{c}_{i}^{*}-\mathbf{Z}_{i} \alpha
\end{array}\right]\left[\begin{array}{ll}
\mathbf{u}_{i}^{\prime} & \left.\left(\mathbf{c}_{i}^{*}-\mathbf{Z}_{i} \alpha\right)^{\prime}\right]
\end{array}\right] \\
& \bar{v}=\overline{\mathbf{H}}_{v}^{-1}\left[\underline{\mathbf{H}}_{v} \underline{\boldsymbol{v}}+\sum_{i=1}^{n}\left(\begin{array}{c}
\mathbf{u}_{i} \\
\mathbf{c}_{i}^{*}-\mathbf{Z}_{i} \alpha
\end{array}\right) m_{i}^{*}\right] .
\end{aligned}
$$

Convergence of the algorithm. The conditions set for by Roberts and Smith (1994) for the posterior distribution to be the unique stationary distribution for a Gibbs sampling algorithm, described as Gibbs sampler convergence condition 2 in Geweke (1996) are satisfied. The key technical condition is that the support of the posterior distribution in latent variables and parameters is connected and upper semicontinuous.

Performance of the Gibbs sampling algorithm in practice. Collect the parameter vectors in the single vector $\theta^{\prime}=\left(\alpha^{\prime}, \beta^{\prime}, \gamma^{\prime}, \delta^{\prime}\right)$ and the data in the single vector $\mathbf{y}$. A posterior moment can then be expressed $\mathrm{E}[\mathrm{g}(\theta) \mid \mathbf{y}]$ for the appropriate function of interest $\mathrm{g}$. The Gibbs sampling algorithm produces serially correlated draws $\theta^{(1)}, \ldots, \theta^{(M)}$ from the posterior distribution. Hence $\mathrm{g}\left(\theta^{(1)}\right), \ldots, \mathrm{g}\left(\theta^{(M)}\right)$ is a sequence of draws from the posterior distribution of $\mathrm{g}(\theta)$. The numerical approximation of $\bar{g}=\mathrm{E}[\mathrm{g}(\theta) \mid \mathbf{y}]$ is $\bar{g}_{M}=M^{-1} \sum_{m=1}^{M} \mathrm{~g}\left(\theta^{(m)}\right)$. Standard methods for serially correlated time series (Geweke (1999), Section 3.7) then produce a consistent (in $M$ ) approximation of $\tilde{\sigma}^{2}=\lim _{M \rightarrow \infty} M \mathrm{E}\left(\bar{g}^{(M)}-\bar{g}\right)^{2}$. [Expectation in the latter expression is with respect to the Markov chain that defines the Gibbs sampling algorithm.]

The efficiency of any Markov chain Monte Carlo (MCMC) algorithm can be evaluated by comparing $\tilde{\sigma}^{2}$ with the posterior variance of $\left.\mathrm{g}(\theta), \sigma^{2}=\mathrm{E}\{\mathrm{g}(\theta)-\bar{g}]^{2} \mid \mathbf{y}\right\}$. If the algorithm produced i.i.d. draws from the posterior distribution, then $\tilde{\sigma}^{2}=\sigma^{2}$. More generally, the relative 
numerical efficiency of any MCMC algorithm is $R N E=\sigma^{2} / \tilde{\sigma}^{2}$. Numerical approximations of $\bar{g}$ based on $M$ iterations of the algorithm will have the same accuracy as $R N E \cdot M$ iterations from a hypothetical algorithm that made i.i.d. drawings directly from the posterior distribution. The ratio of the standard error of approximation $\left(\tilde{\sigma}^{2} / M\right)^{1 / 2}$, to the posterior standard deviation $\sigma$, is $(R N E \cdot M)^{1 / 2}$. For any given posterior distribution and MCMC algorithm, RNE will be different for different functions of interest.

In the selection model in this study, the most important functions of interest are the hospital quality probits $q_{j}$ and hospital severity correlations $\rho_{j}$. In the probit model the most important functions of interest or the hospital quality probits $q_{j}^{*}$. The Gibbs sampling algorithm exhibits strong serial correlation, and for this reason little is lost by using every 20 'th draw (after discarding the first 15,000). This leaves 1900 draws for the selection model and 1750 draws for the probit model.

Table A-1 provides some information on the relative numerical efficiency of these draws. Efficiency in the selection model Gibbs sampling algorithm is much lower than that of the probit model Gibbs sampling algorithm. This is due mainly to the fact that there are 117 latent variables corresponding to each observation in the selection model, whereas the probit model has only one. The median $R N E$ of 1.39 for the probit model $q_{j}^{*}$ implies an accuracy of approximation the same as if $1750 \times 1.39=2432$ draws had been made directly from the posterior distribution. The median RNE of .0112 for the selection model $q_{j}$ implies an accuracy of approximation the same as if 22.1 draws had been made from the posterior. For the $\rho_{j}$ in the selection model the accuracy is equivalent to that of only 8.1 independent draws from the posterior distribution.

To put these assessments in further context, note that there are two sources of uncertainty about any function of interest: that contained in the posterior distribution, and that arising from the finite number of iterations of the MCMC algorithm. The fraction of variance due to the latter is $(1+R N E \cdot M)^{-1}$. At the median, this fraction is $0.04 \%$ for the $q_{j}^{*}, 4.5 \%$ for the $q_{j}$, and $11.0 \%$ for the $\rho_{j}$.

Computational time. Using an IBM 332Mhz 604e processor and ESSL matrix computation routines, the computational time per iteration was approximately 6 minutes. This processor is comparable to a Pentium III 600. We then used an IBM SP supercomputer with Silver nodes, each of which has the $604 \mathrm{e}$ processor as its base, in order to compute each iteration in parallel. Two steps were very parallelizable: the $c_{i j}^{*}$ can be computed in parallel for each individual, and the matrix multiplications necessary to compute the conditional posterior of $\alpha$ can also be broken up by individual. We were able to reduce the computation time close to proportionally to the number of processors that we used. For instance, the algorithm took 100 seconds per iteration 
with 4 processors, 60 seconds per iteration with 8 processors ad 33 second per iteration with 20 processors. Thus, computation time for 50,000 iterations with 20 processors is roughly 19 days. 


\section{Table A-1}

Some relative numerical efficiencies of the algorithm for various parameters

$\begin{array}{lccc}\quad \text { Parameters: } & q_{j}(117 \text { parameters }) & \rho_{j}(116 \text { parameters }) & q_{j}^{*}(117 \text { parameters }) \\ \text { Average } & .0259 & .0046 & 1.75 \\ \text { Lowest } & .0041 & .0028 & 0.32 \\ \text { Third quartile } & .0073 & .0034 & 1.03 \\ \text { Median } & .0112 & .0041 & 1.39 \\ \text { First quartile } & .0189 & .0051 & 2.05 \\ \text { Highest } & .5422 & .0153 & 5.47\end{array}$

